\title{
Iterated relative recursive enumerability ${ }^{\star}$
}

\author{
Peter A. Cholak ${ }^{1, \star \star}$, Peter G. Hinman ${ }^{2}$ \\ 1 Department of Mathematics, University of Notre Dame, Mail Distribution Center, \\ Notre Dame, IN 46556-5683, USA \\ Phone: (219) 631-6507 \\ e-mail: cholaketuring.math.nd.edu \\ 2 Department of Mathematics, University of Michigan, Ann Arbor, MI 48109-1003, USA \\ Phone: (313) 763-3138 \\ e-mail: pgheumich.edu
}

Received November 2, 1993 / in revised form Jnly 15, 1994

\begin{abstract}
A result of Soare and Stob asserts that for any non-recursive r.e. set $C$, there exists a r.e.[C] set $A$ such that $A \oplus C$ is not of r.e. degree. A set $Y$ is called [of] $m$-REA ( $m$-REA[C]) [degree] iff it is [Turing equivalent to] the result of applying $m$-many iterated 'hops' to the empty set (to $C$ ), where a hop is any function of the form $X \mapsto X \oplus W_{e}^{X}$. The cited result is the special case $m=0, n=1$ of our Theorem. For $m=0,1$, and any $(m+1)$-REA set $C$, if $C$ is not of $m$-REA degree, then for all $n$ there exists a $n$-r.e.[C] set $A$ such that $A \oplus C$ is not of $(m+n)$-REA degree. We conjecture that this holds also for $m \geq 2$.
\end{abstract}

\section{Introduction and summary}

This paper is in the recent tradition of studying sets (of natural numbers) and (Turing) degrees which although not recursively enumerable (r.e.) are closely related to r.e. sets and degrees. Our starting point is the following pair of results.

1.1 Theorem(Cooper-Epstein-Lachlan, unpublished) There exists a 2-r.e. set which is not of r.e. degree.

1.2 Theorem[SoSt] For any non-recursive r.e. set $C$, there exists an REA/C] set which is not of r.e. degree.

We recall that a set $A$ is 2-r.e. (or d.r.e) iff there exist r.e. sets $A_{0}$ and $A_{1}$ such that $A$ is their set difference, $A=A_{0} \sim A_{1}$. A set $A$ is r.e.[C] (recursively enumerable in $C$ ) iff for some r.e. set $U$,

$$
A=U^{C}=\{x: \exists y\langle x, C\lceil y\rangle \in U\} .
$$

\footnotetext{
* German speakers should not be unduely influenced by the acronym for this title

** Partially supported by an NSF Postdoctoral Fellowship and the US Army Research Office through the Mathematical Sciences Institute of Cornell University
} 
Here $C\lceil y$ denotes the number which codes the initial segment of the characteristic function of $C$ of length $y$. We say that $\langle x, C\lceil y\rangle$ is an axiom that is satisfied by $C$ and witnesses that $x \in A$. $A$ is REA[C] (recursively enumerable in and above $C$ ) iff $A$ is r.e. $[C]$ and also $C \leq_{T} A$. Note that if $A$ is r.e.[C], then the recursive join $A \oplus C$ is $\operatorname{REA}[C]$. Hence, up to degree, every $\operatorname{REA}[C]$ set is of the form $C \oplus U^{C}$ for some r.e. set $U$.

We shall review in the next section the proofs of these theorems. The basic idea is that in either case the set $A$ may "change its mind" twice about the membership status of any number $x$; this flexibility enables a diagonalization procedure for constructing $A$ not Turing equivalent with any r.e. set.

Our goal here is to establish some natural generalizations of these results. The notion of a set which may "change its mind" any finite number of times is well-known; we give the relativized version:

1.3 Definition. For any sets $A$ and $C$ and any $n$,

(i) $A$ is 0-r.e.[C] iff $A \leq_{T} C$;

(ii) $A$ is $(n+1)$-r.e.[C] iff $A=A_{0} \sim B$ for some r.e.[C]

set $A_{0}$ and some $n$-r.e. $[C]$ set $B$;

(iii) $A$ is $n$-r.e. iff $A$ is $n$-r.e.[ $\emptyset]$.

For this and other properties defined below, a degree has the property iff it contains at least one set with the property. Now a first candidate for a generalization of Theorems 1.1 and 1.2 is

1.4 Theorem. For all $n$,

(i) there exists an $(n+1)$-r.e. set which is not of n-r.e. degree;

(ii) for any non-recursive r.e. set $C$, there exists a set $A$ which is n-r.e.[C] such that $A \oplus C$ is not of n-r.e. degree.

A strengthening of part (i) is proved in [JoSh2] and will be discussed further below; the analogous strengthening of part (ii) will follow from some of our results below. In both cases the strengthening consists in replacing $n$-r.e. by $n$-REA:

1.5 Definition. For any sets $A$ and $C$ and all $n$,

(i) $A$ is $0-\operatorname{REA}[C]$ iff $A \equiv_{T} C$;

(ii) $A$ is $(n+1)$-REA[C] iff $A$ is $\operatorname{REA}[B]$ for some $B$ which is $n$-REA $[C]$;

(iii) $A$ is $n$-REA iff $A$ is $n$-REA[ $\emptyset]$.

This notion was introduced and studied by Jockusch and Shore [JoSh2], who proved in particular

1.6 Theorem. [JoSh2, Theorem 1.4] For all sets $A$ and $C$ and all $n$,

(i) if $A$ is n-r.e.[C], then $A \oplus C$ is of n-REA[C] degree;

(ii) there exists an $(n+1)$-r.e. [C] set $A$ such that $A \oplus C$ is not of n-REAIC] degree.

These clearly imply part (i) of Theorem 1.4 and suggest the following strengthening of (ii), which we shall prove in Sect. 3:

1.7 Theorem. For any non-recursive r.e. set $C$ and any $n$, there exists an n-r.e. $[C]$ set $A$ such that $A \oplus C$ is not of n-REA degree. Hence, there exists a set which is $n-R E A[C]$ but not of $n-R E A$ degree.

Of course, Theorem 1.2 is exactly the case $n=1$ of this. Soare [So, p.116] introduced the term hop for any mapping of the form $C \mapsto C \oplus U^{C}$ for an r.e. set $U$. The Turing jump $C \mapsto C^{\prime}$ is (up to degree) a hop and for every hop, $C \oplus U^{C} \leq_{T} C^{\prime}$. 
If we represent a given hop by $\stackrel{\forall}{\longrightarrow}$ and one we construct by $\stackrel{\exists}{\longrightarrow}$, then the second clause of this theorem may be represented by the diagram

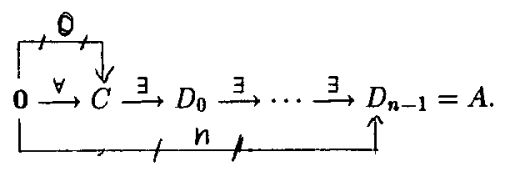

In words, for any $C$ which is reachable from 0 by one hop but not fewer, there exists a set $A$ reachable from $C$ in $n$ hops but not reachable from 0 in $n$ hops. This perspective suggests the following question - if for some $m>0, C$ is reachable from $\mathbf{0}$ in $m+\mathbf{1}$ hops but not in $m$ hops, is there a set reachable from $C$ in $n$ hops which is not reachable from $\mathbf{0}$ in $m+n$ hops. More precisely,

1.8 Conjecture. For any set $C$ and any $m$ and $n$, if $C$ is $(m+1)-R E A$ but not of $m-R E A$ degree, then there exists an n-r.e. $[C]$ set $A$ such that $A \oplus C$ is not of $(m+n)-R E A$ degree. Hence there exists a set which is $n$-REAICl, hence $(m+n+1)-R E A$, but not of $(m+n)-R E A$ degree.

The second clause may be represented by the diagram:

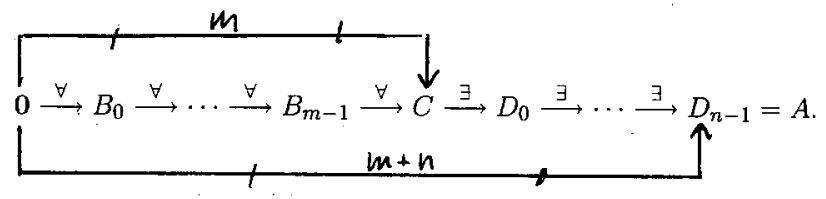

At one point in the work on this paper we believed that we had proved this conjecture and announced it in [ChHi]. Unfortunately, our "proof" contained a gap that we have been unable to fill and we are currently able to prove only

1.9 Main Theorem. The Conjecture holds for all $n$ for $m=0$ and $m=1$.

We shall refer to Case $(m, n)$ of the Conjecture with the obvious meaning. Note that Theorem 1.2 is the case $(0,1)$, Theorem 1.7 comprises all cases $(0, n)$ and that all cases $(m, 0)$ are trivial. Where not otherwise specified, our notation conforms with that of $[\mathrm{So}$.

\section{Background}

To facilitate understanding of the somewhat complex proofs in the following sections, we sketch here in a compatible notation and style proofs of some of the known results mentioned in Sect. 1. We begin with a

Proof (of Theorem 1.1). To construct a 2-r.e. set $A=A_{0} \sim A_{1}$ which is not of r.e. degree, it suffices to satisfy all requirements of the form

$$
A \neq \Phi^{E} \quad \text { or } \quad E \neq \Psi^{A}
$$

where $\Phi$ and $\Psi$ are any recursive functionals and $E$ is any r.e. set. We describe a strategy to satisfy a single instance of (1) while imposing at most finitely much 
restraint; it is then a standard exercise in the finite injury priority method to combine these strategies for all instances of (1).

Suppose that (1) fails, so that in particular for any fixed number $x$

$$
A(x)=\Phi^{E}(x) \quad \text { and } \quad E\left\lceil u(x)=\Psi^{A}\lceil u(x),\right.
$$

where $u(x)$ is the $E$-use of the computation $\Phi^{E}(x)$. Let $v(x)$ denote the maximum of $x+1$ and the $A$-use of $\dot{\Psi}^{A}\left\lceil u(x)\right.$ - that is, the combined $A$-use of $\Psi^{A}(y)$ for $y \leq x$. The key to the strategy is that the potential failure of (1) can be recognized at a finite stage of the construction and evasive action taken to avoid it. Let $\phi(s, x)$ denote the condition

$$
A_{s}(x)=\Phi_{s}^{E_{s}}(x) \quad \text { and } \quad E_{s}\left\lceil u(s, x)=\Psi_{s}^{A_{s}}\lceil u(s, x),\right.
$$

where $u(s, x)$ is the $E_{s}$-use of the computation $\Phi_{s}^{E_{s}}(x) ; \Phi_{s}, \Psi_{s}$ and $E_{s}$ refer to standard enumerations, and $A_{s}$ is the $s$-th stage of the set we are constructing: $A_{s}=A_{0, s} \sim$ $A_{1, s}$. Let $v(s, x)$ denote the maximum of $x+1$ and the $A_{s}$-use of the computations $\Psi_{s}^{A_{s}}\lceil u(s, x)$. By increasing them if necessary, we may assume that $u(s, x)$ and $v(s, x)$ are monotone non-decreasing with respect to both $s$ and $x$.

We say that $\phi(s, x)$ holds correctly iff $\phi(s, x)$ holds, $E_{s}\lceil u(s, x)=E\lceil u(s, x)$, and $A_{s} \uparrow v(s, x)=A \uparrow v(s, x)$. Since $A_{0}, A_{1}$, and $E$ are r.e. sets, if (1) fails, then for all sufficiently large $s$ we have $E_{s}\left\lceil u(x)=E\left\lceil u(x)\right.\right.$, and $A_{s}\lceil v(x)=A\lceil v(x)$, from which it follows that $u(s, x)=u(x), v(s, x)=v(x)$ and $\phi(s, x)$ holds correctly. Thus,

(1.1) (Recognition) if (1) fails, then for all $x$ and all sufficiently large $s, \phi(s, x)$ holds correctly.

Note that $\phi(s, x)$ alone is a recursive condition, whereas its correctness is not. In the construction we shall search for stages where $\phi(s, x)$ holds without regard for correctness; in fact, it is crucial that some instances are not correct.

The strategy now goes as follows. Choose $x \notin A_{0}, A_{1}$ and wait for a stage $s_{0}$ such that $\phi\left(s_{0}, x\right)$. If there is none, then (1) is satisfied by (1.1); otherwise enumerate $x$ into $A_{0, s_{0}+1}$ and restrain $A_{s_{0}+1}\left\lceil v\left(s_{0}, x\right)\right.$ - that is, ensure that no $x^{\prime}<v\left(s_{0}, x\right)$ is enumerated into either $A_{0}$ or $A_{1}$ at any stage $s>s_{0}+1$ at which this restraint is in effect. Recall that $x<v\left(s_{0}, x\right)$. Now wait for a stage $s_{1}>s_{0}$ such that $\phi\left(s_{1}, x\right)$. If there is none, then (1) is satisfied by (1.1); otherwise enumerate $x$ into $A_{1, s_{1}+1}$ and continue the restraint of $A\left\lceil v\left(s_{0}, x\right)\right.$. Let $u_{0}=u\left(s_{0}, x\right)$ and $v_{0}=v\left(s_{0}, x\right)$. Since

$$
\Phi_{s_{0}}^{E_{s_{0}}}(x)=A_{s_{0}}(x)=0 \neq 1=A_{s_{1}}(x)=\Phi_{s_{1}}^{E_{s_{1}}}(x),
$$

it follows that $E_{s_{0}} \uparrow u_{0} \neq E_{s_{1}} \uparrow u_{0}$. But for all $t>s_{1}$ clearly $A_{t}(x)=0=A_{s_{0}}(x)$, whence by the restraint imposed, $A_{t} \uparrow v_{0}=A_{s_{0}} \uparrow v_{0}$ so that

$$
E_{t}\left\lceil u_{0} \neq E_{s_{0}}\left\lceil u_{0}=\Psi_{s_{0}}^{A_{s_{0}}}\left\lceil u_{0}=\Psi_{t}^{A_{t}}\left\lceil u_{0} .\right.\right.\right.\right.
$$

The inequality holds because $E$ is an r.e. set; once it changes on an initial segment it will never revert to its previous value. Now we have also that for all $t>s_{1}$,

$$
E_{t}\left\lceil u(t, x) \neq \Psi_{t}^{A_{t}}\right\rceil u(t, x)
$$

so that $\phi(t, x)$ does not hold and again (1) is satisfied by (1.1).

The main difficulty with the generalization to Theorem 1.6(ii) (here with $C=\emptyset$ ) lies in finding the appropriate condition $\phi(s, x)$ such that the analogue of (1.1) holds. 
For example, to construct a 3-r.e. set $A=A_{0} \sim\left(A_{1} \sim A_{2}\right)$ not of 2-REA degree it is necessary to satisfy requirements

$$
A \neq \Phi^{E \oplus W^{E}} \quad \text { or } \quad E \neq \Psi^{A} \quad \text { or } \quad W^{E} \neq \Theta^{A}
$$

for all recursive functionals $\Phi, \Psi$, and $\Theta$ and all r.e. sets $E$ and $W$. In order to recognize the potential failure of (1) at a stage $s$ for the purpose of taking action to avoid this failure, we need appropriate approximations to all the sets and functionals involved. The key feature in the proof of Theorem 1.1 above is that under the hypothesis that (1) (of that proof) fails, the standard approximations $A_{s}, E_{s}, \Phi_{s}^{E_{s}}$ and $\Psi_{s}^{A_{s}}$ all converge on initial segments to $A, E, \Phi^{E}$ and $\Psi^{A}$, respectively. In the generalized context, however, the standard approximation $W_{s}^{E_{s}}$ does not in general converge to $W^{E}$, since it may happen that for some $y$ there are infinitely many $s$ and $z_{s}$ such that $\left\langle y, E_{s}\left\lceil z_{s}\right\rangle \in W_{s}\right.$, so $y \in W_{s}^{E_{s}}$, but for all such $s, E_{s}\left\lceil z_{s} \neq E\left\lceil z_{s}\right.\right.$ and $y \notin W^{E}$.

The solution to this problem found by Jockusch and Shore is to use $\Theta_{s}^{A_{s}}$ itself as the approximation to $W^{E}$ and to include in $\phi(s, x)$ conditions which ensure that there is sufficient coherence between the approximation and the set approximated. We give below the details for the general case.

2.1 Theorem. [JoSh2, Proposition 1.7] For all n, there exists an $(n+1)$-r.e. set which is not of n-REA degree.

Proof. Any $n$-REA set $E^{n}$ is determined by a sequence $E^{1}, W_{1}, \ldots, W_{n-1}$ of r.e. sets. For $1 \leq i<n$, we define recursively

$$
F^{1}=E^{1}, \quad F^{i+1}=W_{i}^{E^{i}}, \quad \text { and } \quad E^{i+1}=E^{i} \oplus F^{i+1} .
$$

We aim to construct r.e. sets $A_{0}, \ldots, A_{n}$ such that

$$
A=A_{0} \sim\left(A_{1} \sim\left(A_{2} \sim \cdots \sim\left(A_{n-1} \sim A_{n}\right) \cdots\right)\right)
$$

satisfies all requirements of the form

$$
A \neq \Phi^{E^{n}} \quad \text { or } \quad \bigvee_{1 \leq i \leq n}\left[F^{i} \neq \Theta_{i}^{A}\right]
$$

Suppose that (1) fails so that for each $x$,

$$
A(x)=\Phi^{E^{n}}(x) \quad \text { and } \quad \bigwedge_{1 \leq i \leq n}\left[F ^ { i } \left\lceilw^{i}(x)=\Theta_{i}^{A}\left\lceil w^{i}(x)\right]\right.\right.
$$

where $u^{n}(x)$ is the $E^{n}$-use of the computation $\Phi^{E^{n}}(x)$, and recursively, for $1 \leq i<n$, $u^{i}(x)$ and $w^{i}(x)$ are chosen minimal such that for all $y$,

$$
\begin{array}{ll}
2 y<u^{i+1}(x) & \Rightarrow y<u^{i}(x) \\
2 y+1<u^{i+1}(x) & \Rightarrow y<w^{i+1}(x) \\
y \in F^{i+1} \mid w^{i+1}(x) & \Rightarrow\left(\exists z<u^{i}(x)\right)\left\langle y, E^{i} \mid z\right\rangle \in W_{i} \\
w^{1}(x)=u^{1}(x) . &
\end{array}
$$

These parameters, together with the analogous ones at stage $s$, are chosen precisely to guarantee the Recognition and Positive Change properties below. Let $v(x)$ be the maximum of $x+1$ and the total $A$-use for all computations for which $A$ is an oracle. 
For any $s$, let $E_{s}^{1}, W_{i, s}$ and $\Theta_{i, s}^{A_{s}}$ denote the standard approximation to $E^{1}, W_{i}$ and $\Theta_{i}^{A}$ at stage $s$, respectively. For $1 \leq i<n$, set

$$
F_{s}^{i}=\Theta_{i, s}^{A_{s}} \quad \text { and } \quad E_{s}^{i+1}=E_{s}^{i} \oplus F_{s}^{i+1} .
$$

This is, of course, an abuse of notation, since $\Theta_{i, s}^{A_{s}}(y)$ is defined for only finitely many $y$ so $F_{s}^{i}$ is not a characteristic function. In practice we shall always refer to $F_{s}^{i} \uparrow w$, for some $w$, and we interpret this usage to imply that $\Theta_{i, s}^{A_{s}}(y)$ is defined for all $y<w$.

Let $\phi(s, x)$ denote the condition that

$$
A_{s}(x)=\Phi_{s}^{E_{s}^{n}}(x)
$$

and for $1 \leq i \leq n$ there exist $u^{i}(s, x), v(s, x)$, and $w^{i}(s, x)$, such that $u^{n}(s, x)$ is the $E_{s}^{n}$-use of this computation and

$$
\begin{aligned}
& 2 y<u^{i+1}(s, x) \quad \Longrightarrow y<u^{i}(s, x) \\
& 2 y+1<u^{i+1}(s, x) \quad \Longrightarrow y<w^{i+1}(s, x) \\
& y \in F_{s}^{i+1} \mid w^{i+1}(s, x) \Longleftrightarrow\left(\exists z<u^{i}(s, x)\right)\left\langle y, E_{s}^{i}\lceil z\rangle \in W_{i, s}\right. \\
& F_{s}^{1}\left\lceil w^{1}(s, x)=E_{s}^{1} \mid w^{1}(s, x)\right. \\
& w^{1}(s, x) \quad=u^{1}(s, x),
\end{aligned}
$$

and $v(s, x)$ is the maximum of $x+1$ and the total $A_{s}$-use for all computations for which $A_{s}$ is an oracle. As in the preceding proof we may assume that all of these functions are monotone non-decreasing with respect to $s$ and $x$. We say that $\phi(s, x)$ holds correctly iff $\phi(s, x)$ holds; for $1 \leq i \leq n, E_{s}^{i}\left\lceil u^{i}(s, x)=E^{i} \uparrow u^{i}(s, x)\right.$; and $A_{s}\lceil v(s, x)=A\lceil v(s, x)$.

We need first to establish two key properties of these approximations:

(1.1) (Recognition) if (1) fails, then for all $x$ and all sufficiently large stages $s, \phi(s, x)$ holds correctly;

(1.2) (Positive Change) for all $x$ and all $s<t$ such that both $\phi(s, x)$ and $\phi(t, x)$,

(a) for $1 \leq i<n$,

$$
E_{s}^{i}\left\lceil u^{i}(s, x)=E_{t}^{i}\left\lceilu ^ { i } ( s , x ) \Longrightarrow F _ { s } ^ { i + 1 } \left\lceilw ^ { i + 1 } ( s , x ) \subseteq F _ { t } ^ { i + 1 } \left\lceil w^{i+1}(s, x) ;\right.\right.\right.\right.
$$

(b) for $1 \leq i \leq n$,

$E_{s}^{i}\left\lceil u^{i}(s, x) \neq E_{t}^{i}\left\lceil u^{i}(s, x) \Longrightarrow\right.\right.$ for some $1 \leq j \leq i, F_{s}^{j} \uparrow w^{j}(s, x) \subset F_{t}^{j}\left\lceil w^{j}(s, x)\right.$.

To prove (1.1), fix $x$ and suppose that (1) fails at $x$ with $u^{i}(x), v(x)$, and $w^{i}(x)$ defined as above. Choose $s^{1}$ large enough so that for all $s \geq s^{1}$,

$$
A_{s}\left\lceil v(x)=A\left\lceilv ( x ) = \Phi _ { s } ^ { E ^ { n } } ( x ) \quad \text { and } \quad F _ { s } ^ { 1 } \left\lceil u^{1}(x)=E_{s}^{1}\left\lceil u^{1}(x)=E^{1}\left\lceil u^{1}(x)\right.\right.\right.\right.\right.
$$

This is possible since these approximations converge. Let $w^{1}(s, x)=u^{1}(s, x)=u^{1}(x)$. Next choose $s^{2} \geq s^{1}$ such that for all $s \geq s^{2}, F_{s}^{2}\left\lceil w^{2}(x)=F^{2}\left\lceil w^{2}(x)\right.\right.$, and for all $\mathrm{y}$,

$$
y \in F^{2}\left\lceilw ^ { 2 } ( x ) \quad \Longrightarrow \quad ( \exists z < u ^ { 1 } ( x ) ) \left[\left\langle y, E^{1}\lceil z\rangle \in W_{1, s}\right] .\right.\right.
$$

Then $E_{s}^{2} \uparrow u^{2}(x)=E^{2} \uparrow u^{2}(x)$, and if we set $w^{2}(s, x)=w^{2}(x)$, we have for all $y$, 


$$
\begin{aligned}
y \in F_{s}^{2}\left\lceil w^{2}(s, x)\right. & \Longleftrightarrow y \in F^{2}\left\lceil w^{2}(x)\right. \\
& \Longleftrightarrow\left(\exists z<u^{1}(x)\right)\left\langle y, E^{1}\lceil z\rangle \in W_{1}\right. \\
& \Longleftrightarrow\left(\exists z<u^{1}(s, x)\right)\left\langle y, E_{s}^{1}\lceil z\rangle \in W_{1, s},\right.
\end{aligned}
$$

as required for $\phi(s, x)$ to hold.

Continuing recursively, we obtain finally $s^{n}$ such that for all $s \geq s^{n}, E_{s}^{n}\left\lceil u^{n}(x)=\right.$ $E^{n} \uparrow u^{n}(x)$, so

$$
A_{s}(x)=A(x)=\Phi^{E^{n}}(x)=\Phi_{s}^{E_{s}^{n}}(x),
$$

and thus $\phi(s, x)$ holds correctly.

(1.2)(a) is immediate from the definition of $\phi(s, x)$ : if $y \in F_{s}^{i+1}\left\lceil w^{i+1}(s, x)\right.$, then for some $z<u^{i}(s, x),\left\langle y, E_{s}^{i} \mid z\right\rangle \in W_{i, s} \subseteq W_{i, t}$. By hypothesis, $E_{s}^{i}\left\lceil z=E_{t}^{i} \mid z\right.$, so also $y \in F_{t}^{i+1}$.

(1.2)(b) is immediate for $i=1$ and we proceed by induction; assume the result for $i$ and $E_{s}^{i+1}\left\lceil u^{i+1}(s, x) \neq E_{t}^{i+1}\left\lceil u^{i+1}(s, x)\right.\right.$. If $E_{s}^{i}\left\lceil u^{i}(s, x) \neq E_{t}^{i} \uparrow u^{i}(s, x)\right.$, then the conclusion for some $j \leq i$ follows from the induction hypothesis. Otherwise, by (a), $F_{s}^{i+1}\left\lceil w^{i+1}(s, x) \subseteq F_{t}^{i+1}\left\lceil w^{i+1}(s, x)\right.\right.$ and for some $y$ with $2 y+1<u^{i+1}(s, x)$ we have $F_{s}^{i+1}(y) \neq F_{t}^{i+1}(y)$. Thus $y<w^{i}(s, x)$ and this inclusion is proper, so the conclusion holds for $j=i+1$.

Now, the strategy for satisfying a single requirement (1) while imposing finite restraint is as follows. Choose a witness $x$ which belongs to none of the sets $A_{0}, \ldots, A_{n}$ and wait for a stage $s_{0}$ such that $\phi\left(s_{0}, x\right)$. If there is none, (1) is satisfied by (1.1); otherwise, enumerate $x$ into $A_{0, s_{0}+1}$, restrain $A_{s_{0}+1} \mid v\left(s_{0}, x\right)$, and wait for a stage $s_{1}>s_{0}$ such that $\phi\left(s_{1}, x\right)$. If there is none, (1) is satisfied by (1.1); otherwise enumerate $x$ into $A_{1, s_{1}+1}$, restrain $A_{s_{1}+1}\left\lceil v\left(s_{1}, x\right)\right.$, and wait for a stage $s_{2}>s_{1}$ such that $\phi\left(s_{2}, x\right)$. If (1) is not satisfied at any stage of this process by (1.1), then we generate a sequence $s_{0}<s_{1}<\ldots<s_{n}$ such that for $j \leq n, \phi\left(s_{j}, x\right)$.

Let $u_{j}^{i}=u^{i}\left(s_{j}, x\right), v_{j}=v\left(s_{j}, x\right)$, and $w_{j}^{i}=w^{i}\left(s_{j}, x\right)$. We establish first the following fact: for all $1 \leq i<n$ and $j<(n-1)$, (*)

$$
E_{s_{j}}^{i+1}\left\lceil u_{j}^{i+1} \neq E_{s_{j+1}}^{i+1}\left\lceil u_{j}^{i+1} \Rightarrow \text { for some } h \leq i, \quad E_{s_{j+1}}^{h}\left\lceil u_{j+1}^{h} \neq E_{s_{j+2}}^{h}\left\lceil u_{j+1}^{h}\right. \text {. }\right.\right.\right.
$$

By the hypothesis of this implication and (1.2)(b), for some $h \leq i, F_{s_{j}}^{h+1}\left\lceil w_{j}^{h+1} \subset\right.$ $F_{s_{j+1}}^{h+1} \mid w_{j}^{h+1}$. On the other hand, since enumerating $x$ in both $A_{j}$ and $A_{j+1}$ has no net effect, by the restraint imposed we have $A_{s_{j}}\left\lceil v_{j}=A_{s_{j+2}}\left\lceil v_{j}\right.\right.$, so

$$
F_{s_{j}}^{h+1}\left\lceil w_{j}^{h+1}=\Theta_{h+1, s_{j}}^{A_{s_{j}}}\left\lceil w_{j}^{h+1}=\Theta_{h+1, s_{j+2}}^{A_{s_{j+2}}}\left\lceil w_{j}^{h+1}=F_{s_{j+2}}^{h+1}\left\lceil w_{j}^{h+1} .\right.\right.\right.\right.
$$

and it follows that

$$
F_{s_{j+1}}^{h+1}\left\lceil w_{j}^{h+1} \nsubseteq F_{s_{j+2}}^{h+1}\right\rceil w_{j}^{h+1} \quad \text { hence also } \quad F_{s_{j+1}}^{h+1}\left\lceil w_{j+1}^{h+1} \nsubseteq F_{s_{j+2}}^{h+1}\right\rceil w_{j+1}^{h+1} .
$$

The desired conclusion follows by (1.2)(a).

Now

$$
\Phi_{s_{0}}^{E_{s_{0}}^{n}}(x)=A_{s_{0}}(x)=0 \neq 1=A_{s_{1}}(x)=\Phi_{s_{1}}^{E_{s_{1}}^{n}}(x),
$$

and thus $E_{s_{0}}^{n_{b}}\left\lceil u_{0}^{n} \neq E_{s_{1}}^{n}\left\lceil u_{0}^{n}\right.\right.$. Using (*) it follows by induction that there exist $n=h_{0}>\cdots>h_{n-1}$ such that for $j<n, E_{s_{j}}^{h_{j}}\left\lceil u_{j}^{h_{j}} \neq E_{s_{j+1}}^{h_{j}} \uparrow u_{j}^{h_{j}}\right.$, so in fact $h_{j}=n-j$ and in particular, $E_{s_{n-1}}^{1}\left\lceil u_{n-1}^{1} \neq E_{s_{n}}^{1} \uparrow u_{n-1}^{1}\right.$. But then for all $t>s_{n}$ we have $A_{t}\left\lceil v_{n}=A_{s_{n-1}}\left\lceil v_{n}\right.\right.$ and thus 


$$
E_{t}^{1} \uparrow u_{n-1}^{1} \neq E_{s_{n-1}}^{1} \uparrow u_{n-1}^{1}=\Theta_{1, s_{n-1}}^{A_{s_{n-1}}}\left\lceil u_{n-1}^{1}=\Theta_{1, t}^{A_{t}}\left\lceil u_{n-1}^{1}\right.\right.
$$

and hence $\phi(t, x)$ does not hold and (1) is satisfied by (1.1).

Theorem 1.6(i) is less germane to our methods here, but the following short proof seems not to have appeared in print, so we include it.

2.2 Lemma. For any sets $A$ and $C$ and all $n$, if $A$ is $(n+1)-r . e .[C]$, then $A \oplus C$ is of $R E A[D \oplus C]$ degree for some n-r.e.[C] set $D$.

Proof. Let $A$ be $(n+1)$-r.e.[C], so $A=A_{0} \sim B$ for some r.e.[C] set $A_{0}$ and some $n$ r.e. $[C]$ set $B$. Fix a $C$-recursive function $f$ such that $A_{0}=\operatorname{Im}(f)$ and let $D=f^{-1}(B)$. Easily $D$ is $n$-r.e. [C] and it suffices to observe that

$$
D \leq_{T} A, \quad \text { since for all } x, \quad x \in D \Longleftrightarrow f(x) \notin A ;
$$

and

$A$ is r.e. $[D], \quad$ since for all $x, \quad x \in A \Longleftrightarrow \exists y[y \notin D \wedge f(y)=x]$.

2.3 Corollary. For any sets $A$ and $C$ and all $n$, if $A$ is n-r.e. $[C]$, then $A \oplus C$ is of $n$-REA[C] degree.

Proof. We proceed by induction. For $n=0$, the result is clear. If $A$ is $(n+1)$-r.e.[C], choose $D$ as in the Lemma. By the induction hypothesis, $D \oplus C \equiv_{T} E$ for some $n$-REA $[C]$ set $E$, and $A \oplus C$ is of REA $[E]$-degree, hence of $(n+1)$-REA[C] degree.

Before we turn to an exposition of the proof of Theorem 1.2, we have to dispose of a technical point, which is nonetheless of great importance in that proof and others to follow. It concerns the fact that the results relative to an arbitrary non-recursive set are necessarily non-uniform - that is, an index for the set constructed cannot be computed recursively from the indices of the given sets. To formulate this precisely, fix an enumeration $\left\langle W_{e}: e \in \omega\right\rangle$ of the r.e. sets and set $X^{\langle e\rangle}=X \oplus W_{e}^{X}, X^{\langle\rangle}=X$, and

$$
X^{\left\langle e_{0}, \ldots, e_{m}\right\rangle}=\left(X^{\left\langle e_{0}, \ldots, e_{m-1}\right\rangle}\right)^{\left\langle e_{m}\right\rangle} .
$$

We call a sequence $\left(e_{0}, \ldots, e_{m}\right)$ of (indices of) $m+1$ many hops non-degenerate iff $\emptyset\left\langle e_{0}, \ldots, e_{m}\right\rangle$ is not of $m$-REA degree. The second clause of our Conjecture 1.8 may be reformulated as follows: for all $m, n$, and $b_{0}, \ldots, b_{m}$, if $\left(b_{0}, \ldots, b_{m}\right)$ is non-degenerate, then there exist $d_{0}, \ldots, d_{n-1}$ such that $\left(b_{0}, \ldots, b_{m}, d_{0}, \ldots, d_{n-1}\right)$ is non-degenerate. Then we have the following generalization of [SoSt, Corollary 4.3].

2.4 Proposition. For all $m$ and $n$, there do not exist recursive functions $g_{0}, \ldots, g_{n}$ such that for all $b_{0}, \ldots, b_{m}$, if $\left(b_{0}, \ldots, b_{m}\right)$ is non-degenerate, then also

$$
\left(b_{0}, \ldots, b_{m}, g_{0}\left(b_{0}, \ldots, b_{m}\right), \ldots, g_{n}\left(b_{0}, \ldots, b_{m}\right)\right)
$$

is non-degenerate.

Proof. By the relativized version of [JoSh1, Theorem 3.1] there exists a recursive function $h$ such that for all sets $X$ and indices $e$,

$$
X<_{T} X^{\langle h(e)\rangle} \quad \text { and } \quad X^{\langle h(e), e\rangle} \equiv_{T} X^{\prime} .
$$


Now, given $m, n$, and recursive $g_{0}, \ldots, g_{n}$, let $b_{0}=\cdots=b_{m-1}$ be indices for the Turing jump and choose $b_{m}$ by the Recursion Theorem such that for all $X$,

$$
W_{h\left(g_{0}\left(b_{0}, \ldots, b_{m-1}, b_{m}\right)\right)}^{X}=W_{b_{m}}^{X} .
$$

Then by the first property of $h$, for any $X, X<_{T} X^{\left\langle b_{m}\right\rangle}$, so

$$
\emptyset^{\left\langle b_{0}, \ldots, b_{m-1}\right\rangle}=\emptyset^{(m)}<_{T} \emptyset^{\left\langle b_{0}, \ldots, b_{m-1}, b_{m}\right\rangle},
$$

and thus $\emptyset^{\left\langle b_{0}, \ldots, b_{m-1}, b_{m}\right\rangle}$ is not of $m$-REA degree - that is, $\left(b_{0}, \ldots, b_{m}\right)$ is nondegenerate. But by the second property of $h$,

$$
\emptyset^{\left\langle b_{0}, \ldots, b_{m-1}, b_{m}, g_{0}\left(b_{0}, \ldots, b_{m}\right)\right\rangle} \equiv T\left(\emptyset^{\left\langle b_{0}, \ldots, b_{m-1}\right\rangle}\right)^{\prime},
$$

is of $m+1-$ REA degree, from which it follows that

$$
\emptyset^{\left\langle b_{0}, \ldots, b_{m}, g_{0}\left(b_{0}, \ldots, b_{m}\right), \ldots, g_{n}\left(b_{0}, \ldots, b_{m}\right)\right\rangle}
$$

is of $m+n+1$-REA degree - that is,

$$
\left(b_{0}, \ldots, b_{m}, g_{0}\left(b_{0}, \ldots, b_{m}\right), \ldots, g_{n}\left(b_{0}, \ldots, b_{m}\right)\right)
$$

is degenerate.

Finally, we sketch

Proof of Theorem 1.2. Let $C$ be a given non-recursive r.e. set. We aim to construct an r.e. set $U$, such that if $A=U^{C}$, then $A \oplus C$ is not of r.e. degree. In view of the preceding proposition, we shall in fact construct two r.e. sets $U_{0}$ and $U_{1}$ such that $A_{k}=U_{k}^{C}(k=0,1)$ satisfy the family of requirements

$$
\bigvee_{k=0,1}\left[A_{k} \neq \Phi_{k}^{E_{k}} \quad \text { or } \quad E_{k} \neq \Psi_{k}^{A_{k} \oplus C}\right]
$$

for all sextuples $\left(\Phi_{0}, \Phi_{1}, \Psi_{0}, \Psi_{1}, E_{0}, E_{1}\right)$ of four recursive functionals and two r.e. sets. It follows that at least one of the sets $A_{0} \oplus C, A_{1} \oplus C$ is not of r.e. degree as desired; the non-uniformity arises in that we can not determine effectively which one.

If (1) fails, then for any $x$,

$$
\bigwedge_{k=0,1}\left[A _ { k } \left\lceilx+1=\Phi_{k}^{E_{k}}\left\lceilx + 1 \quad \text { and } \quad E _ { k } \left\lceil u(x)=\Psi_{k}^{A_{k} \oplus C}\lceil u(x)]\right.\right.\right.\right.
$$

where $u(x)$ is the combined use of $\Phi_{0}^{E_{0}}(y)$ and $\Phi_{1}^{E_{1}}(y)$ for $y \leq x$. The difficulty in recognizing this potential failure is somewhat similar to that in Theorem 2.1 - the standard approximations for the r.e. sets $E_{k}$ and $C$ converge, but the approximations $A_{k, s}=U_{k, s}^{C_{s}}$ do not generally converge to $A_{k}$. In this case we shall invoke a standard device, the method of true stages. Fix an enumeration $\left\langle C_{s}: s \in \omega\right\rangle$ of $C$ such that $C_{s+1} \sim C_{s} \neq \emptyset$ for all $s$ and let $C_{s+1}$ denote the smallest element of this set. A stage $s$ is $C$-true iff $C_{s}\left\lceil c_{s}=C \uparrow c_{s}\right.$. It is clear that there are infinitely many $C$-true stages and we shall arrange the construction to ensure that at any $C$-true stage $s$, we have $A_{k, s} \subseteq A_{k}$ so that for any $v$, for sufficiently large $C$-true stages, $A_{k, s}\left\lceil v=A_{k}\lceil v\right.$ - in other words, $A_{k, s}$ converges to $A_{k}$ on $C$-true stages.

Let $\phi(x, s)$ denote the condition 


$$
\bigwedge_{k=0,1}\left[A _ { k , s } \left\lceilx+1=\Phi_{k, s}^{E_{k, s}}\left\lceilx + 1 \quad \text { and } \quad E _ { k , s } \left\lceil u(s, x)=\Psi_{k, s}^{A_{k, s} \oplus C_{s}}\lceil u(s, x)]\right.\right.\right.\right.
$$

where $u(s, x)$ is the combined use of the computations $\Phi_{k, s}^{E_{k, s}}(y)$ for $k=0,1$ and $y \leq x$. We assume that $u(s, x)$ is monotone non-decreasing in both $s$ and $x$. Note that we now require agreement of $A_{k, s}$ and $\Phi_{k, s}^{E_{k, s}}$ for all $y \leq x$ instead of merely at $x$; this is a minor change required by the use of multiple witnesses as described below. Let $v(s, x)$ denote the maximum of $x+1$ and the $A_{k, s}$ and $C_{s}$ uses in these computations and say that $\phi(s, x)$ holds correctly iff for $k=0,1, E_{k, s}\left|u(s, x)=E_{k}\right| u(s, x)$, $A_{k, s}\left\lceil v(s, x)=A_{k}\left\lceil v(s, x)\right.\right.$, and $C_{s}\lceil v(s, x)=C \mid v(s, x)$. Then much as above, we have

(1.1) (Recognition) if (1) fails, then for all $x$ and all sufficiently large $C$-true stages $s, \phi(s, x)$ holds correctly.

The mechanism for ensuring convergence on $C$-true stages is simple: at each stage $t+1$ of the construction we enumerate into $U_{k, t+1}$ certain axioms $\left\langle x, C_{t} \mid v\right\rangle$, with $v \leq c_{t+1}$; thus $x \in A_{k, t+1}$. If $c_{s}<v$ for some $s>t$, then $C_{s} \uparrow v \neq C_{t} \uparrow v$, so generally $x \notin A_{k, s}$. If $s$ is $C$-true and $x \in A_{k, s}$, then $v \leq c_{s}$, so $C_{t}\left\lceil v=C_{s} \uparrow v=C\lceil v\right.$, and thus also $x \in A_{k}$.

The strategy for satisfying a single instance of (1) is complicated in two ways (relative to Theorem 1.1) by the replacement of the 2-r.e. set $A$ by $A \oplus C$ with $A=U^{C}$. First, the set $A \oplus C$ is subject to unpredictable changes due to changes in $C$. Second, at the point in the proof of Theorem 1.1 when we enumerate $x$ into $A_{1}$ to remove it from $A$, we can now only wait for a change in some $C \uparrow v$, which may or may not ever occur, to remove $x$ from $A=U^{C}$.

The solution to both of these problems consists in assigning to (1) not a single witness but a (potentially) infinite sequence $\left\langle x_{l}: l \in \omega\right\rangle$ of witnesses. Success with any one of the witnesses $x_{l}$ suffices to satisfy (1) and we show that failure of all the witnesses leads to an algorithm for computing $C$, contrary to its assumed non-recursiveness. Roughly, the failure of a witness $x_{l-1}$ is due to the fact that the corresponding $C \uparrow v_{l}$ does not change to remove $x_{l-1}$ from $A$ and thus $C \uparrow v_{l}$ has its final value at a predictable stage.

Before giving the formal construction, we describe it informally. We define the sequence of witnesses as we go along; $x_{l}$ will be a candidate for enumeration into $A_{\pi(l)}$, where $\pi(l)=0$, if $l$ is even; 1 , if $l$ is odd. Let $x_{0}=0$. We take no action until we arrive at a stage $s_{0}$ for which $\phi\left(s_{0}, x_{0}\right)$ holds. If there is no such stage, then (1) holds by (1.1) (since there are infinitely many $C$-true stages); otherwise we define $x_{1}$ larger than any number mentioned so far, in particular, $x_{1}>v_{0}:=v\left(s_{0}, x_{0}\right)$, restrain $A_{0, s_{0}+1}\left\lceil v_{0}\right.$, and wait for a stage $s_{1}>s_{0}$ at which $\phi\left(s_{1}, x_{1}\right)$ holds. If there is none, then again (1) holds by (1.1); otherwise enumerate $x_{0}$ into $A_{0, s_{1}+1}$ with use $v_{1}=v\left(s_{1}, x_{1}\right)$ - that is, enumerate the axiom $\left\langle x_{0}, C_{s_{1}}\left\lceil v_{1}\right\rangle\right.$ into $U_{0, s_{1}+1}$ - restrain $A_{1, s_{1}+1}\left\lceil v_{1}\right.$, and choose $x_{2}>v_{1}$. Similarly, we wait for a stage $s_{2}>s_{1}$ at which $\phi\left(s_{2}, x_{2}\right)$ holes, enumerate $x_{1}$ into $A_{1, s_{2}+1}$ with use $v_{2}$, restrain $A_{0, s_{2}+1} \uparrow v_{2}$, and choose $x_{3}>v_{2}$. At $s_{3}, x_{2}$ is enumerated into $A_{0}$, and so on.

The following diagram may be helpful in following this argument. 


$$
\begin{aligned}
& \begin{array}{lllllll}
0 & . & \frac{s_{0}}{\rho=0} & \frac{s_{0}+1}{\rho=1} & \cdots & \frac{s_{1}}{\rho=1} & \frac{s_{1}+1}{\rho=2}
\end{array}
\end{aligned}
$$

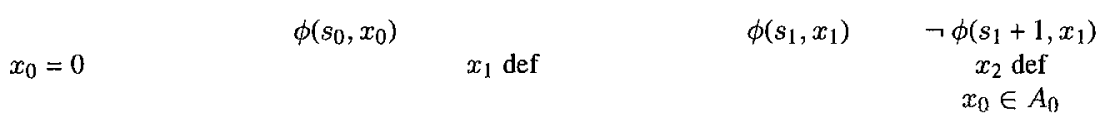

$$
\begin{aligned}
& \begin{array}{ccccc}
\frac{s_{2}}{\rho=2} & \frac{s_{2}+1}{\rho=3} & \ldots & \frac{s_{l}}{\rho=l} & \frac{s_{l}+1}{\rho=l+1} \\
\phi\left(s_{2}, x_{2}\right) & \neg \phi\left(s_{2}+1, x_{2}\right) & & \phi\left(s_{l}, x_{l}\right) & \neg \phi\left(s_{l}+1, x_{l}\right) \\
& x_{3} \operatorname{def} & & & x_{l+1} \operatorname{def} \\
& x_{1} \in A_{1} & & & x_{l-1} \in A_{\pi(l-1)}
\end{array}
\end{aligned}
$$

Each instance of $\phi\left(s_{l}, x_{l}\right)$ is dependent on the value of $C_{s_{l}}\left\lceil v_{l}\right.$; if this is not the correct value $C \uparrow v_{l}$, the agreement recorded by $\phi\left(s_{l}, x_{l}\right)$ may be destroyed at some later stage. Let us assume temporarily that this never happens, although in the end we shall show that it must happen.

Let $u_{l}=u\left(s_{l}, x_{l}\right)$; by our conventions, $u_{l}$ is monotone non-decreasing in $l$. Since

$$
A_{0, s_{0}} \uparrow v_{0}=A_{0, s_{1}} \uparrow v_{0}, \quad \phi\left(s_{0}, x_{0}\right), \quad \text { and } \phi\left(s_{1}, x_{1}\right),
$$

we have

$$
E_{0, s_{0}}\left\lceil u_{0}=\Psi_{0, s_{0}}^{A_{0, s_{0}} \oplus C_{s_{0}}}\left\lceil u_{0}=\Psi_{0, s_{1}}^{A_{0_{1} s_{1}} \oplus C_{s_{1}}}\left\lceil u_{0}=E_{0, s_{1}}\left\lceil u_{0}\right.\right.\right.\right.
$$

and $u\left(s_{1}, x_{0}\right)=u_{0}$. On the other hand, since $\phi\left(s_{1}, x_{1}\right), \phi\left(s_{2}, x_{2}\right)$, and $x_{0}<x_{1}<x_{2}$, we have

$$
\Phi_{0}^{E_{0, s_{1}}}\left(x_{0}\right)=A_{0, s_{1}}\left(x_{0}\right)=0 \neq 1=A_{0, s_{2}}\left(x_{0}\right)=\Phi_{0, s_{2}}^{E_{0, s_{2}}}\left(x_{0}\right) .
$$

Hence $E_{0, s_{1}}\left\lceil u_{0} \neq E_{0, s_{2}}\left\lceil u_{0}\right.\right.$, and if we set

$$
t_{0}=\text { least } t>s_{0}\left[E _ { 0 , s _ { 0 } } \left\lceilu_{0} \neq E_{0, t}\left\lceil u_{0}\right]\right.\right. \text {, }
$$

then $s_{1}<t_{0} \leq s_{2}$. Furthermore,

$$
s_{1}=\text { least } s<t_{0}\left[\phi\left(s, x_{1}\right) \wedge C_{s} \uparrow v_{1}=C_{t_{0}} \uparrow v_{1}\right] .
$$

This means that from $s_{0}$ we can effectively determine $s_{1}$. Extending this argument we obtain an algorithm for the function $l \mapsto s_{l}$, which is therefore recursive. But clearly $v_{l}>l$, so by our temporary assumption that $C_{s_{l}}\left\lceil v_{l}=C\left\lceil v_{l}\right.\right.$ we have $C(l)=C_{s_{l}}(l)$ and we conclude, contrary to hypothesis, that $C$ is recursive.

Thus our temporary hypothesis is untenable, and it is exactly this fact that guarantees that (1) is satisfied. To see how this works, suppose that for $j \leq l$, $C_{s_{j}}\left\lceil v_{j}=C\left\lceil v_{j}\right.\right.$ but $C_{s_{l+1}}\left\lceil v_{l+1} \neq C\left\lceil v_{l+1}\right.\right.$. Since $x_{l}$ is enumerated into $A_{\pi(l), s_{l+1}+1}$ with the axiom $\left\langle x_{l}, C_{s_{l+1}} \uparrow v_{l+1}\right\rangle$, if at some later $t>s_{l+1}, C_{t} \uparrow v_{l+1} \neq C_{s_{l+1}} \uparrow v_{l+1}$, then $x_{l} \notin A_{\pi(l), t}$ so by the restraint imposed, $A_{\pi(l), t} \uparrow v_{l}=A_{\pi(l), s_{l}} \uparrow v_{l}$. Now if also $E_{\pi(l), t}\left\lceil u_{l} \neq E_{\pi(l), s_{l}}\left\lceil u_{l}\right.\right.$, then for all $s \geq t$ we have

$$
E_{\pi(l), s}\left\lceil u_{l} \neq E_{\pi(l), s_{l}}\left\lceil u_{l}=\Psi_{\pi(l), s_{l}}^{A_{\pi(l), s_{l}} \oplus C_{s_{l}}}\left\lceil u_{l}=\Psi_{\pi(l), s}^{A_{\pi(l), s} \oplus C_{s}}\left\lceil u_{l},\right.\right.\right.\right.
$$

so $\phi\left(s, x_{l}\right)$ fails and (1) is satisfied by (1.1). On the other hand, if $E_{\pi(t), t} \mid u_{l}=$ $E_{\pi(l), s_{l}}\left\lceil u_{l}\right.$, then there may be a stage $s_{l+1}^{\prime} \geq t$ such that $\phi\left(s_{l+1}^{\prime}, x_{l+1}\right)$ with use $v_{l+1}^{\prime}=v\left(s_{l+1}^{\prime}, x_{l+1}\right)$ - whence also $\phi\left(s_{l+1}^{\prime}, x_{l}\right)$. At this point we re-enumerate $x_{l}$ into $A_{\pi(l), s_{l+1}^{\prime}+1}$ with use $v_{l+1}^{\prime}$, redefine $x_{l+2}>v_{l+1}^{\prime}$, and wait for a stage $s_{l+2}$ such that 
$\phi\left(s_{l+2}, x_{l+2}\right)$. Again $C_{s_{l+1}^{\prime}} \mid v_{l+1}^{\prime}$ may or may not be correct. Thus there are three possibilities:

(a) for some $l$, from some point on, $\phi\left(s, x_{l}\right)$ never holds;

(b) for some $l$, for infinitely many $s, \phi\left(s, x_{l}\right)$ holds with use $v\left(s, x_{l}\right)$, but $C_{s}$ । $v\left(s, x_{l}\right) \neq C\left\lceil v\left(s, x_{l}\right)\right.$;

(c) for all $l$ there is eventually a stage $\bar{s}_{l}$ such that $\phi\left(\bar{s}_{l}, x_{l}\right)$ with use $\bar{v}_{l}=v\left(\bar{s}_{l}, x_{l}\right)$ and $C_{\bar{s}_{l}} \uparrow \bar{v}_{l}=C \nmid \bar{v}_{l}$.

In cases (a) and (b), requirement (1) is satisfied by (1.1); note that in case (b) it is the correctness of $\phi\left(s, x_{1}\right)$ which is infinitely often violated. By a variant of the argument given above, case (c) cannot hold, and thus (1) is satisfied.

The rest of the machinery of the proof consists in bookkeeping to keep track of the situation at stage $s$. We set $\rho(s)=l$ if at stage $s$ are defined witnesses $x_{j}(s)$ for $j \leq l$ and uses $u_{j}(s)$ and $v_{j}(s)$ for $j<l$. Here $x_{j}(s)$ is the current value of $x_{j}$ and the uses are those of the most recent instance of $\phi\left(\cdot, x_{j}\right)$. At stage $s$ we take action only if either case 1: $c_{s+1}<v_{j}(s)$ for some $j<l$, or case 2: $\phi\left(s, x_{l}(s)\right)$ via computations with use $\leq c_{s+1}$. If neither ever occurs, then $x_{l}=x_{l}(s)$ is a successful witness. If case 1 occurs, then the earlier agreements up to $x_{j}(s), \ldots, x_{l}(s)$ have been injured and we reduce our list of potential witnesses to $x_{0}(s+1), \ldots, x_{j}(s+1)$; the witnesses $x_{j+1}(s), \ldots, x_{l}(s)$ are discarded with new values possibly to be chosen at later stages. If case 2 occurs, then we set $u_{l}(s+1)$ and $v_{l}(s+1)$ to be the associated uses, choose a new witness $x_{l+1}(s+1)$ larger than any number mentioned so far, and (if $l>0$ ) enumerate $x_{l-1}(s)$ into $A_{\pi(l-1), s+1}$ with use $v_{l}(s+1)$. The formal proof that $(1)$ is satisfied now proceeds by establishing the following two facts:

(1.3) If (1) fails, then $\liminf _{s} \rho(s)=\infty$.

(1.4) If $\liminf _{s} \rho(s)=\infty$, then $C$ is recursive, contrary to hypothesis.

Now if we set

$$
s_{l}=\text { least } s(\forall t>s)[\rho(t) \geq l+1],
$$

then the condition $\liminf _{s} \rho(s)=\infty$ is exactly the condition that all $s_{l}$ exist, which is essentially the temporary hypothesis of our sketch above. Note that when $s_{l}$ exists, $C\left\lceil v_{l}\right.$ has its final value at stage $s_{l}$, and to compute $C$ it suffices to compute the function $l \mapsto s_{l}$.

We now give the precise construction and a series of lemmas which formalize the preceding argument. Set $\rho(0)=0$ and $x_{0}(0)=x_{0}=0$. At stage $s+1$ we have one of three cases as follows. Any parameter not mentioned is assigned the same value at $s+1$ as at $s$.

Case 1. If $\rho(s)>0$ and for some (least) $k<\rho(s), \quad c_{s+1}<v_{k}(s)$, then $\rho(s+1):=k$; $u_{j}(s+1)$, and $v_{j}(s+1)$ are undefined for all $j \geq k$; and $x_{j}(s+1)$ is undefined for all $j>k$;

Case 2. otherwise, if $\rho(s)=l$ and $\phi\left(s, x_{l}(s)\right)$ holds, with associated uses $u\left(s, x_{l}(s)\right)$ and $v\left(s, x_{l}(s)\right) \leq c_{s+1}$, then

(a) $\rho(s+1):=l+1, u_{l}(s+1)=u\left(s, x_{l}(s)\right)$, and $v_{l}(s+1)=v\left(s, x_{l}(s)\right)$;

(b) if $l>0$, then $x_{l-1}(s)$ is enumerated into $A_{\pi(l-1), s+1}$ with use $v_{l}(s+1)$;

(c) $x_{l+1}(s+1)$ is chosen greater than any number used so far;

Case 3. otherwise, $\rho(s+1):=\rho(s)$.

We note first 
(2) For all $s, i$, and $j$,

(a) $x_{j}(s)$ is defined for (exactly) $j \leq \rho(s) ; \quad u_{j}(s)$ and $v_{j}(s)$ are defined for (exactly) $j \leq \rho(s)-1$;

(b) $i<j \leq \rho(s) \Longrightarrow v_{i}(s) \leq x_{j}(s)$;

(c) $j<\rho(s+1) \Longrightarrow v_{j}(s) \leq c_{s+1}$;

(d) for (exactly) $j \leq \rho(s)-2, \quad x_{j}(s) \in A_{\pi(j), s}$ with use $\leq c_{s}$;

(e) for all $t<s$, if $x_{j}(t)$ is defined but $x_{j}(s)$ is either undefined or $\neq x_{j}(t)$, then for all $s^{\prime} \geq s, x_{j}(t) \notin A_{\pi(j), s^{\prime}}$. In particular, this holds when $\rho(s)<j \leq \rho(t)$.

Proof. Parts (a)-(d) are straightforward to verify by induction. for (e), suppose that $x_{j}(t)$ is enumerated into $A_{\pi(j), t}$ with use $v_{j+1}(t)$. In either of the cases of the hypothesis, for some $t^{\prime}$ with $t \leq t^{\prime}<s, x_{j}$ becomes undefined at stage $t^{\prime}+1$ because $c_{t^{\prime}+1}<v_{j}\left(t^{\prime}\right)$. If $v_{j+1}\left(t^{\prime}\right)=v_{j+1}(t)$, then also $c_{t^{\prime}+1}<v_{j+1}(t)$ and $x_{j}(t)$ is removed from $A_{\pi(j), t^{\prime}+1}$; otherwise it was already removed at an earlier stage. Now the number $x_{j}(t)$ will never again be used as a witness so is never again enumerated into $A_{\pi(j)}$.

(3)

For each $l \geq 0$, if $s_{l}$ exists, then

(a) $\rho\left(s_{l}\right)=\bar{l}, \quad \phi\left(s_{l}, x_{l}\right)$, and $\rho\left(s_{l}+1\right)=l+1$;

(b) for all $s>s_{l}, \quad \rho(s) \geq l+1$;

(c) for all $j \leq l, x_{j+1}(t), u_{j}(t)$ and $v_{j}(t)$ have the same values for all $t>s_{l}$ (which we denote by $x_{j+1}, u_{j}$, and $v_{j}$ );

(d) $C_{s_{l}}\left\lceil v_{l}=C\left\lceil v_{l}\right.\right.$;

(e) for all $j \leq(l-2), \quad x_{j} \in A_{\pi(j), s}$ for all $s \geq s_{l}$;

(f) if $l \geq 1$, then $x_{i-1} \notin A_{\pi(l-1), s_{i}}$, but for all $s>s_{l}, x_{l-1} \in A_{\pi(l-1), s}$.

Proof. (a) is immediate from the minimality of $s_{l}$ and (b) is merely a restatement of the definition. Now by the condition $v_{l}(s+1) \leq c_{s+1}$ in case $2, C_{s_{l}}\left\lceil v_{l}(s+1)=C_{s_{l}+1} \mid\right.$ $v_{l}(s+1)$. Then for (c) and (d) we prove by induction that for all $t>s_{l}, x_{j+1}(t), u_{j}(t)$, and $v_{j}(t)$ have the same values as at stage $s_{l}+1$ and that $C_{t} \backslash v_{l}(t)=C_{t+1}\left\lceil v_{l}(t)\right.$. The key point is that case 1 will never apply at any stage $t+1>s_{l}+1$ with $k \leq l$, since then $\rho(t+1)=k \leq l$ contrary to the definition of $s_{l}$. Parts (e) and (f) follow from (a), (b), (d) and (2)(e).

We have also the converse to $(3)(d)$

(4) For any $l$ and $s$, if $\rho(s) \geq l+1$ and $C_{s} \uparrow v_{l}(s)=C \uparrow v_{l}(s)$, then $s_{l}$ exists and $s>s_{l}$.

Proof. Here, again, the point is that case 1 will never apply at any stage $t \geq s+1$ with $k \leq l$

(5) For each $l \geq 0$, if $s_{l+1}$ exists, then $A_{\pi(l), s_{l+1}}\left\lceil v_{l}=A_{\pi(l), s_{l}} \uparrow v_{l}\right.$.

Proof. It suffices to verify that for all $j$ and all $t \leq s_{l+1}$ such that $x_{j}(t)<v_{l}$ we have

$$
A_{\pi(l), s_{l}}\left(x_{j}(t)\right)=A_{\pi(l), s_{l+1}}\left(x_{j}(t)\right) .
$$

For $h>l+1$, this follows from (2)(e): $x_{j}(t)$ belongs to neither set. No value $x_{l+1}(t)$ belongs to either set because $\pi(l+1) \neq \pi(l)$. For $j \leq l, x_{j}(s)=x_{j}:=x_{j}\left(s_{l}\right)$ for all $s \geq s_{l}$, so again by (2)(e), if for some $t<s_{l}, x_{j}(t) \neq x_{j}$, then $x_{j}(t)$ belongs to neither set. Hence we are left with the the numbers $x_{j}$ for $j \leq l$. Of these, only those such that $\pi(j)=\pi(l)-$ that is, $h \equiv l \quad(\bmod 2)-$ belong to either set and by $(3)(e)$ and $(f)$, for such $j$, 


$$
\begin{gathered}
h \leq l-2 \quad \Longrightarrow \quad\left(\forall s \geq s_{l}\right) x_{j} \in A_{\pi(l), s} \\
x_{l} \notin A_{\pi(l), s_{l}} \quad \text { and } \quad x_{l} \notin A_{\pi(l), s_{l+1}} . \quad \square
\end{gathered}
$$

(6) For each $l \geq 0$,

(a) if $s_{l+1}$ exists, then, $E_{\pi(l), s_{l+1}} \uparrow u_{l}=E_{\pi(l), s_{l}}\left\lceil u_{l}\right.$;

(b) if $s_{l+2}$ exists, then, $\left(\exists s>s_{l}\right)\left[E_{\pi(l), s}\left\lceil u_{l} \neq E_{\pi(l), s_{l}}\left\lceil u_{l}\right]\right.\right.$, and if $t_{l}$ denotes the least such $s$, then $s_{l+1}<t_{l}$.

Proof. For (a), since both $\phi\left(s_{l+1}, x_{l}\right)$ and $\phi\left(s_{l}, x_{l}\right)$ hold, by (5) and the definition of $v_{l}$,

$$
E_{\pi(l), s_{l+1}}\left\lceil u_{l}=\Psi_{\pi(l), s_{l+1}}^{A_{\pi(l), s_{l+1}}} \oplus C_{s_{l+1}}\left\lceil u_{l}=\Psi_{\pi(l), s_{l}}^{A_{\pi(l), s_{l}} \oplus C_{s_{l}}}\left\lceil u_{l}=E_{\pi(l), s_{l}}\left\lceil u_{l}\right.\right.\right.\right.
$$

For (b), since both $\phi\left(s_{l}, x_{l}\right)$ and $\phi\left(s_{l+2}, x_{l}\right)$, if equality holds for $s=s_{l+2}$, we have the contradiction

$$
0=A_{\pi(l), s_{l}}\left(x_{l}\right)=\Phi_{\pi(l), s_{l}}^{E_{\pi(l), s_{l}}}\left(x_{l}\right)=\Phi_{\pi(l), s_{l+2}}^{E_{\pi(l), s_{l+2}}}\left(x_{l}\right)=A_{\pi(l), s_{l+2}}\left(x_{l}\right)=1
$$

The last clause is now immediate from (a).

We can now establish (1.3) and (1.4).

(1.3) If (1) fails, then $\liminf _{s} \rho(s)=\infty$.

Proof. Suppose that (1) fails but $\liminf _{s} \rho(s)=l<\infty$. Thus $s_{l-1}$ exists, but for infinitely many $s, \rho(s)=l$ (so $s_{l}$ does not exist). For $s>s_{l-1}, x_{l}(s)$ has the constant value $x_{l}$. By (1.1) there exists $\bar{s}>s_{l-1}$ such that $\phi\left(\bar{s}, x_{l}\right)$ holds correctly. Then either $\rho(\bar{s}) \geq l+1$ and $\rho(\bar{s}+1) \geq l+1$, or $\rho(\bar{s})=l$, case 2 holds at stage $\bar{s}$ and $\rho(\bar{s}+1)=l+1$. In either case, by (4), $s_{l}$ exists, contrary to hypothesis.

(1.4) Only finitely many $s_{l}$ exist; in other words, $\liminf \operatorname{in}_{s} \rho(s)<\infty$.

Proof. Suppose to the contrary that all $s_{l}$ exist. Then by (6)(b) and (4) for all $l, t_{l}$ exists and

$$
s_{l+1}=\text { least } s<t_{l}\left[\phi\left(s, x_{l+1}\right) \wedge C_{s}\left\lceil v_{l+1}(s)=C_{t_{l}} \uparrow v_{l+1}\left(t_{l}\right)\right]\right.
$$

Since $t_{l}$ and $x_{l+1}$ are recursively calculable from $s_{l}$, this shows that the function $l \mapsto s_{l}$ is recursive. But then since $l<v_{l}, C(l)=C_{s_{l}}(l)$ and $C$ is recursive, contrary to hypothesis.

The remainder of the proof consists in combining the strategies for all requirements (1) on a tree. The outcome of a requirement (1) is the value $l$ of $\liminf _{s} \rho(s)$. We shall not take space here to do this in detail for Theorem 1.2, as the techniques are well illustrated by the Theorem of the next section. 


\section{The main theorem}

In this section we shall give the proof of the cases $(1, n)$ of the Conjecture; the cases $(0, n)$ have a similar but slightly simpler proof which is easily derivable from the one here. That is, we prove

3.1 Theorem. For any set $C$ and any $n$, if $C$ is 2-REA but not of r.e. degree, then there exists an n-r.e.[C] set $A$ such that $A \oplus C$ is not of $(n+1)-R E A$ degree.

Proof. Fix $n \geq 0$ and let $C$ be a fixed 2-REA set, say $C=B \oplus V^{B}$ for r.e. sets $V$ and $B$, such that $C$ is not of r.e. degree. By Proposition 2.4 we must construct at least two $n$-r.e.[C] sets $A_{0}$ and $A_{1}$; in fact, for reasons which will become clear during the proof we construct $(n+2)$ such sets $A_{k}$ for $k<n+2$. For each $k<n+2$ we attempt to ensure that $A_{k} \oplus C$ is not Turing equivalent to any $(n+1)$-REA set $E_{k}^{n+1}$, which is characterized in terms of r.e. sets $E_{k}^{1}, W_{k, 1}, \ldots, W_{k, n}$ by setting for $1 \leq i \leq n$,

$$
F_{k}^{1}=E_{k}^{1}, \quad F_{k}^{i+1}=W_{k, i}^{E^{i}}, \quad E_{k}^{i+1}=E_{k}^{i} \oplus F_{k}^{i+1} .
$$

Each $A_{k}$ will be of the form

$$
A_{k}=A_{k, 0} \sim\left(A_{k, 1} \sim\left(A_{k, 2} \sim \cdots \sim\left(A_{k, n-2} \sim A_{k, n-1}\right) \cdots\right)\right),
$$

where each $A_{k, j}$ is $C$-r.e. To guarantee that at least one such $A_{k}$ is not of $(n+1)$-REA degree, we aim to satisfy all requirements of the form

$$
\bigvee_{k<n+2}\left[A_{k} \neq \Phi_{k}^{E_{k}^{n+1}} \quad \text { or } \quad \bigvee_{1 \leq i \leq n+1}\left[F_{k}^{i} \neq \Theta_{k, i}^{A_{k} \oplus C}\right]\right]
$$

We consider first one such requirement. If it fails, then for all $x$,

$$
\bigwedge_{k<n+2}\left[A_{k} \mid x+1=\Phi_{k}^{E_{k}^{n+1}}\left\lceilx + 1 \text { and } \bigwedge _ { 1 \leq i \leq n + 1 } \left[ F_{k}^{i}\left\lceil w^{i}(x)=\Theta_{k, i}^{A_{k} \oplus C}\left\lceil w^{i}(x)\right]\right]\right.\right.\right.
$$

where $u^{n+1}(x)$ is the combined $E_{k}^{n+1}$-uses of the computations $\Phi_{k}^{E_{k}^{n+1}}(y)$ for $y \leq x$ and $k<n+2$, and for $1 \leq i \leq n, u^{i}(x)$ and $w^{i}(x)$ are chosen minimal such that for all $y$ and all $k<n+2$,

$$
\begin{array}{ll}
2 y<u^{i+1}(x) & \Rightarrow y<u^{i}(x) \\
2 y+1<u^{i+1}(x) & \Longrightarrow y<w^{i+1}(x) \\
y \in F_{k}^{i+1}\left\lceil w^{i+1}(x)\right. & \Rightarrow\left(\exists z<u^{i}(x)\right)\left\langle y, E_{k}^{i}\lceil z\rangle \in W_{i}\right. \\
w^{1}(x)=u^{1}(x) . &
\end{array}
$$

Let $v(x)$ be the maximum of $x+1$ and the $A_{k}$ and $C$-uses for all computations for which $A_{k} \oplus C$ is an oracle.

As in the proof of Theorem 1.2 we shall construct each $C$-r.e. set $A_{k, j}$ as $U_{k, j}^{C}$. However, we note that it suffices for $U_{k, j}$ to be $B$-r.e. rather than outright r.e. This fact allow us to use $B$ freely as an oracle during the construction; in particular we may assume that $C$ is given via a $B$-recursive enumeration $\left\langle C_{s}: s \in \omega\right\rangle$ and in effect treat $C$ as an r.e. set. We define $c_{s}$ and the set of $C$-true stages relative to this enumeration as before. 
Let $A_{k, j, s}=U_{k, j, s}^{C_{s}}, \quad E_{k, s}^{1}, \quad W_{k, i, s}, \quad$ and $\quad \Theta_{k, i, s}^{A_{k, s} \oplus C_{s}}$ be the standard approximations, and for $1 \leq i \leq n$, set

$$
F_{k, s}^{i}=\Theta_{k, i, s}^{A_{k, s} \oplus C_{s}} \quad \text { and } \quad E_{k, s}^{i+1}=E_{k, s}^{i} \oplus F_{k, s}^{i+1},
$$

with the convention as before that when we write $F_{k, s}^{i} \mid w$, we imply that $\Theta_{k, i, s}^{A_{k, s} \oplus C_{s}}(y)$ is defined for all $y<w$. Let $\phi(s, x)$ denote the condition that for all $k<n+2$

$$
A_{k, s} \uparrow x+1=\Phi_{k, s}^{E_{k, s}^{n+1}}\lceil x+1
$$

and for $1 \leq i \leq n+1$ there exist $u^{i}(s, x), v(s, x)$, and $w^{i}(s, x)$ all monotone nondecreasing in both arguments such that $u^{n+1}(s, x)$ is the combined $E_{k, s}^{n+1}$-use of these computations for $y \leq x$, and for $k<n+2$ and all $y$,

$$
\begin{array}{ll}
2 y<u^{i+1}(s, x) & \Longrightarrow y<u^{i}(s, x) \\
2 y+1<u^{i+1}(s, x) & \Longrightarrow y<w^{i+1}(s, x) \\
y \in F_{k, s}^{i+1}\left\lceil w^{i+1}(s, x)\right. & \Longleftrightarrow\left(\exists z<u^{i}(s, x)\right)\left[\left\langle y, E_{k, s}^{i}\lceil z\rangle \in W_{k, i, s}\right]\right. \\
F_{k, s}^{1}\left\lceil w^{1}(s, x)=E_{k, s}^{1}\left\lceil w^{1}(s, x)\right.\right. \\
w^{1}(s, x)=u^{1}(s, x),
\end{array}
$$

and $v(s, x)$ is the maximum of $x+1$ and the combined $A_{k, s}$ and $C_{s}$-uses for all $k<n+2$ and all computations for which $A_{k, s} \oplus C_{s}$ is an oracle. We say that $\phi(s, x)$ holds correctly iff $\phi(s, x)$ holds and for $k<n+2$ and $1 \leq i \leq n+1$, $E_{k, s}^{i}\left\lceil u^{i}(s, x)=E_{k}^{i}\left\lceil u^{i}(s, x)\right.\right.$ and $A_{k, s}\left\lceil v(s, x)=A_{k}\lceil v(s, x)\right.$.

The Recognition and Positive Change properties may be proved exactly as before:

(1.1) (Recognition) if (1) fails, then for all $x$ and all sufficiently large $C$-true stages $s, \phi(s, x)$ holds correctly;

(1.2) (Positive Change) for all $x$, all $k<n+2$, and all $s<t$ such that both $\phi(s, x)$ and $\phi(t, x)$,

(a) for $1 \leq i \leq n$,

$$
E_{k, s}^{i}\left\lceil u^{i}(s, x)=E_{k, t}^{i}\left\lceilu ^ { i } ( s , x ) \Longrightarrow F _ { k , s } ^ { i + 1 } \left\lceilw ^ { i + 1 } ( s , x ) \subseteq F _ { k , t } ^ { i + 1 } \left\lceil w^{i+1}(s, x) ;\right.\right.\right.\right.
$$

(b) for $1 \leq i \leq n+1$,

$$
\begin{aligned}
& E_{k, s}^{i}\left\lceil u^{i}(s, x) \neq E_{k, t}^{i}\left\lceil u^{i}(s, x) \Longrightarrow \text { for some } 1 \leq j \leq i,\right.\right. \\
& F_{k, s}^{j}\left\lceilw ^ { j } ( s , x ) \subset F _ { k , t } ^ { j } \left\lceil w^{j}(s, x) .\right.\right.
\end{aligned}
$$

Before giving the formal construction of sets $A_{k}$ which satisfy a single instance of (1) we describe it informally. As in the proof of Theorem 1.2 we use a potentially infinite sequence $\left\langle x_{l}: l \in \omega\right\rangle$ of witnesses; $x_{l}$ is a witness for $A_{\pi(l)}$, where $\pi(l)=$ $l \bmod (n+2)$. For this sketch let us assume that $n=2$ so that we are constructing 2-r.e[C] sets $A_{k}=A_{k, 0} \sim A_{k, 1}(k<4)$ with the goal that for at least one $k$, $A_{k} \oplus C$ is not of 3-REA degree - in particular, $A_{k} \oplus C$ is not Turing equivalent to $E_{k}^{3}$. Our overall strategy is to show that if (1) fails, then $C$ is Turing equivalent to $B \oplus E_{0}^{1} \oplus \cdots \oplus E_{3}^{1}$ and is hence of r.e. degree, contrary to hypothesis.

Let $x_{0}=0$ and wait for a stage $s_{0}$ such that $\phi\left(s_{0}, x_{0}\right)$ holds. In contrast to the proof of Theorem 1.2 we immediately enumerate $x_{0}$ into $A_{0,0, s_{0}+1}$ with use $v_{0}=v\left(s_{0}, x_{0}\right)$ and as before choose $x_{1}>v_{0}$, restrain $A_{0}\left\lceil v_{0}\right.$, and wait for $s_{1}$ such that $\phi\left(s_{1}, x_{1}\right)$. 
We again make the (untenable) assuption that for each $l, C_{s_{l}} \uparrow v_{l}=C \mid v_{l}$, or more generally that this is true for some eventual $s_{l}$ for each $x_{l}$. At stage $s_{1}$ we enumerate $x_{1}$ into $A_{1,0, s_{1}+1}$ with use $v_{1}$ but we do not enumerate $x_{0}$ into $A_{0,1}$; this delay corresponds to the fact that in the proof of Theorem 1.2 we made no enumeration at stage $s_{0}$. At stage $s_{2}$ where $\phi\left(s_{2}, x_{2}\right)$ holds with use $v_{2}=v\left(s_{2}, x_{2}\right)$ we enumerate $x_{0}$ into $A_{0,1, s_{2}+1}$ and $x_{2}$ into $A_{2,0, s_{2}+1}$, each with use $v_{2}$. At stage $s_{3}$ where $\phi\left(s_{3}, x_{3}\right)$ holds we enumerate $x_{1}$ into $A_{1,1, s_{3}+1}$ and $x_{3}$ into $A_{3,0, s_{3}+1}$ with use $v_{3}$. Finally, at stage $s_{4}$ we return to $A_{0}$ by enumerating $x_{4}$ into $A_{0,0, s_{4}+1}$.

Let $u_{j}^{i}=u^{i}\left(s_{j}, x_{j}\right)$. As in the proof of Theorem 1.2, $E_{0, s_{0}}^{3}\left\lceil u_{0}^{3} \neq E_{0, s_{1}}^{3}\left\lceil u_{0}^{3}\right.\right.$. By the Positive Change property (1.2)(b), for some $1 \leq h \leq 3, F_{0, s_{0}}^{h}\left\lceil w_{0}^{h} \subset F_{0, s_{1}}^{h}\left\lceil w_{0}^{h}\right.\right.$. Since $x_{0}$ is removed from $A_{0}$ at stage $s_{2}+1$, we have $A_{0, s_{3}}\left\lceil v_{0}=A_{0, s_{0}}\left\lceil v_{0}\right.\right.$ and by $\phi\left(s_{3}, x_{0}\right)$,

$$
F_{0, s_{0}}^{h}\left\lceil w_{0}^{h}=\Theta_{0, h, s_{0}}^{A_{0, s_{0}}}\left\lceil w_{0}^{h}=\Theta_{0, h, s_{3}}^{A_{0, s_{3}}}\left\lceil w_{0}^{h}=F_{0, s_{3}}^{h}\left\lceil w_{0}^{h},\right.\right.\right.\right.
$$

so

$$
F_{0, s_{1}}^{h}\left\lceilw _ { 0 } ^ { h } \nsubseteq F _ { 0 , s _ { 3 } } ^ { h } \left\lceilw _ { 0 } ^ { h } , \quad \text { whence also } \quad F _ { 0 , s _ { 1 } } ^ { h } \left\lceilw _ { 1 } ^ { h } \nsubseteq F _ { 0 , s _ { 3 } } ^ { h } \left\lceil w_{1}^{h},\right.\right.\right.\right.
$$

and hence by Positive Change (1.2)(a), $E_{0, s_{1}}^{2}\left\lceil u_{1}^{2} \neq E_{0, s_{3}}^{2} \uparrow u_{1}^{2}\right.$. On the other hand, since we made no enumeration into $A_{0}$ at stage $s_{1}, A_{0, s_{1}} \uparrow v_{1}=A_{0, s_{2}} \uparrow v_{1}$ so $E_{0, s_{1}}^{2}\left\lceil u_{1}^{2}=E_{0, s_{2}}^{2}\left\lceil u_{1}^{2}\right.\right.$.

By analogy with Theorem 1.2, we hope to use this information to compute $s_{2}$ from $s_{1}$ by looking for changes in $E_{0}^{2} \uparrow u_{1}^{2}$. However, $E_{0}^{2}$ is 2-REA rather than r.e. and it is consistent with the above conditions that there are changes between $s_{1}$ and $s_{2}$ which are undone at $s_{2}$. There can be no such change in the r.e. part $E_{0}^{1}\left\lceil u_{1}^{1}\right.$ and any such change in $F_{0}^{2}\left\lceil w_{1}^{2}\right.$ at a stage $s_{1}<t<s_{2}$ must consist in the acquisition of some new elements with axioms satisfied by a value of $E_{0, t}^{1} \uparrow u^{1}\left(t, x_{1}\right)$ which changes by stage $s_{2}$ to remove these elements. In particular, since no correct initial segment of an approximation to an r.e. set ever changes,

$$
E_{0, t}^{1}\left\lceil u^{1}\left(t, x_{1}\right) \neq E_{0}^{1}\left\lceil u^{1}\left(t, x_{1}\right)\right.\right.
$$

Thus, if we set

$$
t_{0}=\text { least } t>s_{1}\left[\phi ( t , x _ { 1 } ) \wedge E _ { 0 , t } ^ { 2 } \left\lceilu_{1}^{2} \neq E_{0, s_{1}}^{2}\left\lceilu _ { 1 } ^ { 2 } \wedge E _ { 0 , t } ^ { 1 } \left\lceil u^{1}\left(t, x_{1}\right)=E_{0}^{1}\left\lceil u^{1}\left(t, x_{1}\right)\right],\right.\right.\right.\right.
$$

then if $t_{0}$ exists, it is greater than $s_{2}$ and we can compute $s_{2}$ from $s_{1}$ by

$$
s_{2}=\text { least } s<t_{0}\left[\phi ( s , x _ { 2 } ) \wedge C _ { s } \left\lceilv\left(s, x_{2}\right)=C_{t_{0}}\left\lceil v\left(s, x_{2}\right)\right] .\right.\right.
$$

This computation uses oracle $B \oplus E_{0}^{1}$; extending this idea gives $C \leq_{T} B \oplus E_{0}^{1} \oplus$ $\cdots \oplus E_{3}^{1}$.

It remains to argue that $t_{0}$ does exist and that $B \oplus E_{0}^{1}$ (and analogously $B \oplus$ $E_{0}^{1} \oplus \cdots \oplus E_{3}^{1}$ ) is recursive in $C$. Both of these arguments depend on the device of constructing four (in general $n+2$ ) rather than only two sets $A_{k}$. Of course, $B \leq_{T} C$ by assumption. The key claim is that $E_{0, s_{3}}^{1} \uparrow u_{3}^{1}=E_{0}^{1} \uparrow u_{3}^{1}$. On the one hand, this guarantees that $t_{0} \leq s_{3}$ and hence exists. On the other, since the function $l \mapsto s_{l}$ is $C$-recursive, this equation describes (the beginning of) an algorithm for computing $E_{0}^{\mathrm{I}}$ from $C$

The claim follows from the fact that for all $t \geq s_{3}, A_{0 . s_{3}}\left\lceil v_{3}=A_{0, t}\left\lceil v_{3}\right.\right.$. This is true because the next witness after $x_{0}$ which is used for $A_{0}$ is $x_{4}$, which is chosen $>v_{3}$, so no number $\leq v_{3}$ is ever again added to $A_{0}$. Of course, the restraint imposed 
ensures that no other requirement does this either, at least on the true path. The claim now follows since for all $l \geq 3$, by $\phi\left(s_{l}, x_{l}\right)$,

$$
E_{0, s_{l}}^{1}\left\lceil u_{3}^{1}=\Theta_{0,1}^{A_{0, s_{l}} \oplus C_{s_{l}}}\left\lceil u_{3}^{1}=\Theta_{0,1}^{A_{0, s_{3}} \oplus C_{s_{3}}}\left\lceil u_{3}^{1}=E_{0, s_{3}}^{1}\left\lceil u_{3}^{1},\right.\right.\right.\right.
$$

and the fact that the approximations $E_{0, s}^{1}$ converge to $E_{0}^{1}$.

The remainder of the notational bookkeeping is very similar to that of Theorem 1.2. We maintain a function $\rho$ such that if $\rho(s)=l$, then there are defined witnesses $x_{j}(s)$ for $j \leq l$ and uses $u_{j}^{i}(s), v_{j}(s)$, and $w_{j}^{i}(s)$ for $j<l$. The proof that $(1)$ is satisfied follows from

(1.3) if (1) fails, then $\lim \inf _{s} \rho(s)=\infty$;

(1.4) if $\liminf _{s} \rho(s)=\infty$, then $C \equiv_{T} B \oplus E_{0}^{1} \oplus \cdots \oplus E_{n-1}^{1}$; hence, $C$ is of r.e. degree, contrary to hypothesis.

We set

$$
s_{l}=\text { least } s(\forall t>s)[\rho(t) \geq l+1] .
$$

By the construction, if $s_{l}$ exists, then $C_{s_{l}} \uparrow v_{l}=C \uparrow v_{l}$, so if all $s_{l}$ exist, then $C$ is recursive in the function $l \mapsto s_{l}$.

Set $\rho(0)=0$ and $x_{0}(0)=0$. At stage $s+1$ we have one of three cases as follows, Any parameter not mentioned is assigned the same value at $s+1$ as at $s$.

Case 1. If $\rho(s)>0$ and for some (least) $h<\rho(s), \quad c_{s+1}<v_{h}(s)$, then $\rho(s+1):=h$; $u_{j}^{i}(s+1), v_{j}(s+1)$, and $w_{j}^{i}(s+1)$ are undefined for all $j \geq h$; and $x_{j}(s+1)$ is undefined for all $j>h$;

Case 2. otherwise, if $\rho(s)=l$ and $\phi\left(s, x_{l}(s)\right)$ holds, with associated uses $u^{i}\left(s, x_{l}(s)\right)$, $w^{i}\left(s, x_{l}(s)\right)$, and $v\left(s, x_{l}(s)\right) \leq c_{s+1}$, then

(a) $\rho(s+1):=l+1, u_{l}^{i}(s+1)=u^{i}\left(s, x_{l}(s)\right), w_{l}^{i}(s+1)=w^{i}\left(s, x_{l}(s)\right)$, and $v_{l}(s+1)=$ $v\left(s, x_{l}(s)\right)$;

(b) for $j<\min \{l, n-1\}, x_{l-j}$ is enumerated into $A_{\pi(l-j), j, s+1}$ with use $v_{l}(s+1)$;

(c) if $l \geq n$, then $x_{l-n}$ is enumerated into $A_{\pi(l-n), n-1, s+1}$ with use $v_{l}(s+1)$;

(d) $x_{l+1}(s+1)$ is chosen greater than any number used so far;

Case 3. otherwise, $\rho(s+1):=\rho(s)$.

The proof now breaks into a series of lemmas as before. In many cases the proofs are straightforward adaptations of the corresponding parts of Theorem 1.2 and we omit them.

(2) For all $s, h, 1 \leq i \leq n$, and $j$,

(a) $x_{j}(s)$ is defined for (exactly) $j \leq \rho(s) ; \quad u_{j}^{i}(s), v_{j}(s)$, and $w_{j}^{i}(s)$ are defined for (exactly) $j \leq \rho(s)-1$;

(b) $h<j \leq \rho(s) \Longrightarrow v_{h}(s) \leq x_{j}(s)$;

(c) $j<\rho(s+1) \Rightarrow v_{j}(s) \leq c_{s+1}$;

(d) for $j<(n-1)$ and $h<\rho(s)-j, \quad x_{h}(s) \in A_{\pi(h), j, s}$ with use $\leq c_{s}$, but for $\rho(s)-j \leq h \leq \rho(s), \quad x_{h} \notin A_{\pi(h), j, s}$;

(e) for $h<\rho(s)-n, \quad x_{h}(s) \in A_{\pi(h), n-1, s}$ with use $\leq c_{s}$, but for $\rho(s)-n \leq h \leq$ $\rho(s), \quad x_{h}(s) \notin A_{\pi(h), n-1, s}$;

(f) for all $t<s$, if $x_{h}(t)$ is defined but $x_{h}(s)$ is either undefined or $\neq x_{h}(t)$, then for all $s^{\prime} \geq s$ and $j<n, x_{h}(t) \notin A_{\pi(h), j, s^{\prime}}$. In particular, this holds when $\rho(s)<h \leq \rho(t)$. 
Proof. For (f), suppose that $x_{h}(t)$ is enumerated into $A_{\pi(h), j, t}$ with use $v_{h+j}(t)$ (for $j<n-1$ ) or $v_{h+j+1}(t)$ (for $j=n-1$ ). In either of the cases of the hypothesis, for some $t^{\prime}$ with $t \leq t^{\prime}<s, x_{h}$ becomes undefined at stage $t^{\prime}+1$ because $c_{t^{\prime}+1}<v_{h}\left(t^{\prime}\right)$. If $v_{h+j(+1)}\left(t^{\prime}\right)=v_{h+j(+1)}(t)$, then also $c_{t^{\prime}+1}<v_{h+j(+1)}(t)$, so $x_{h}(t)$ is removed from $A_{\pi(h), j, t^{\prime}+1}$; otherwise it was already removed at an earlier stage. Now the number $x_{h}(t)$ will never again be used as a witness so it is never again enumerated into $A_{\pi(h), j}$.

(3) For each $l \geq 0$, if $s_{l}$ exists, then

(a) $\rho\left(s_{l}\right)=l, \quad \phi\left(s_{l}, x_{l}\right), \quad$ and $\rho\left(s_{l}+1\right)=l+1$;

(b) for all $s>s_{l}, \quad \rho(s) \geq l+1$;

(c) for all $j \leq l, x_{j+1}(t), u_{j}^{i}(t), w_{j}^{i}(t)$, and $v_{j}(t)$ have the same values for all $t>s_{l}$ (which we denote by $x_{j+1}, u_{j}^{i}$, $w_{j}^{i}$, and $v_{j}$ );

(d) $C_{s_{l}}\left\lceil v_{l}=C\left\lceil v_{l}\right.\right.$

(e) for all $s \geq s_{l}$,

(i) for all $j<(n-1)$ and $h \leq(l-j-1), \quad x_{h} \in A_{\pi(h), j, s}$;

(ii) for all $h \leq(l-n-1), \quad x_{h} \in A_{\pi(h), n-1, s}$;

(f) for all $s>s_{l}$,

(i) for all $j<(n-1), \quad x_{l-j} \notin A_{\pi(l-j), j, s_{l}}, \quad$ but $\quad x_{l-j} \in A_{\pi(l-j), j, s}$;

(ii) $x_{l-n} \notin A_{\pi(l-n), n-1, s_{l}}$, but $\quad x_{l-n} \in A_{\pi(l-n), n-1, s}$.

(4) For any $l$ and $s$, if $\rho(s) \geq l+1$ and $C_{s} \uparrow v_{l}(s)=C \uparrow v_{l}(s)$, then $s_{l}$ exists and $s>s_{l}$.

(5) For each $l \geq 0$, if $s_{l+n+1}$ exists, then

(a) for all $j<(n-2), \quad A_{\pi(l), s_{l+j}}\left\lceil v_{l+j}=A_{\pi(l), s_{l+j+2}}\left\lceil v_{l+j}\right.\right.$;

(b) $A_{\pi(l), s_{l+n-2}}\left\lceil v_{l+n-2}=A_{\pi(l), s_{l+n+1}}\left\lceil v_{l+n-2}\right.\right.$;

(c) $A_{\pi(l), s_{l+n-1}} \uparrow v_{l+n-1}=A_{\pi(l), s_{l+n}}\left\lceil v_{l+n-1}\right.$.

Proof. Consider first (a) and fix $j<(n-2)$. Much as in the corresponding part of the proof of Theorem 1.2 we conclude that for each $h$ and $t \leq s_{t+j+2}$, with the possible exception of $x_{h}:=x_{h}\left(s_{l}\right)$ for $h \leq l+j$ and $h \equiv l \quad(\bmod n+2)$, we have for all $g<n$,

$$
A_{\pi(l), g, s_{l+j}}\left(x_{h}(t)\right)=A_{\pi(l), g, s_{l+j+2}}\left(x_{h}(t)\right) .
$$

Then by (3)(e) and (f), for such $h$,

$$
\begin{array}{cc}
h \leq l-(n+2) \quad \Longrightarrow & (\forall g<n)\left(\forall s \geq s_{l+j}\right) x_{h} \in A_{\pi(l), g, s} ; \\
x_{l} \in A_{\pi(l), g, s_{l+j}} & \text { for exactly } g=0,1, \ldots, j-1 ; \\
& x_{l} \in A_{\pi(l), g, s_{l+j+2}} \quad \text { for exactly } g=0,1, \ldots, j+1 .
\end{array}
$$

Thus, the only permanent change in $A_{\pi(l)}$ between stage $s_{l+j}$ and stage $s_{l+j+2}$ is the enumeration of $x_{l}$ into both $A_{\pi(l), j}$ and $A_{\pi(l), j+1}$, which has no net effect. The proof of (b) is similar; between $s_{l+n-2}$ and $s_{l+n+1}, x_{l}$ is enumerated into $A_{\pi(l), n-2}$ and $A_{\pi(l), n-1}$. For (c), we may show as above that there is no permanent change in any $A_{\pi(l), g}$ between $s_{l+n-1}$ and $s_{l+n}$.

(6) For each $l>0$,

(a) if $s_{l+n+1}$ exists, then for all $j \leq(n-2)$,

$$
E_{\pi(l), s_{l+j}}^{n-j+1}\left\lceil u_{l+j}^{n-j+1} \neq E_{\pi(l), s_{l+j+1}}^{n-j+1}\left\lceil u_{l+j}^{n-j+1}\right.\right.
$$

(b) if $s_{l+n}$ exists, then $E_{\pi(l), s_{l+n-1}}^{2}\left\lceil u_{l+n-1}^{2}=E_{\pi(l), s_{l+n}}^{2}\left\lceil u_{l+n-1}^{2}\right.\right.$; 
(c) if $s_{l+j}$ exists for all $j$, then $E_{\pi(l), s_{l+n+1}}^{1}\left\lceil u_{l+n+1}^{1}=E_{\pi(l)}^{1}\left\lceil u_{l+n+1}^{1}\right.\right.$;

(d) if $s_{l+j}$ exists for all $j$, then

$$
\begin{aligned}
\exists t>s_{l+n-1}\left[\phi\left(t, x_{l+n-1}\right) \wedge\right. & E_{\pi(l), t}^{2}\left\lceil u_{l+n-1}^{2} \neq E_{\pi(l), s_{l+n-1}^{2}}^{2}\left\lceil u_{l+n-1}^{2}\right.\right. \\
& \wedge E_{\pi(l), t}^{1}\left\lceil u^{1}\left(t, x_{l+n-1}\right)=E_{\pi(l)}^{1}\left\lceil u^{1}\left(t, x_{l+n-1}\right)\right],\right.
\end{aligned}
$$

and if $t_{l}$ denotes the least such $t$, then $s_{l+n}<t_{l}$.

Proof. The proof of part (a) is similar to that of the corresponding part in the proof of Theorem 1.2. Using (4) and (5)(a) show first that for $i \leq n$ and $j<(n-2)$,

$$
E_{\pi(l), s_{i+j}}^{i+1}\left\lceil u_{l+j}^{i+1} \neq E_{\pi(b), s_{i+j+1}}^{i+1}\left\lceilu _ { l + j } ^ { i + 1 } \Rightarrow E _ { \pi ( l ) , s _ { i + j + 1 } } ^ { i } \left\lceil u_{l+j+1}^{i} \neq E_{\pi(l), s_{l+j+2}}^{i}\left\lceil u_{l+j+1}^{i} .\right.\right.\right.\right.
$$

Then (a) follows by induction on $j$. Part (b) follows from (5)(c). Part (c) is proved as in the sketch: for all $j \geq(n+1), A_{\pi(l), s_{l+n+1}}\left\lceil v_{l+n+1}=A_{\pi(l), s_{l+j}}\left\lceil v_{l+n+1}\right.\right.$, so

$$
\begin{aligned}
& E_{\pi(l), s_{l+j}}^{1}\left\lceil u_{l+n+1}^{1}=\Theta_{\pi(l), 1, s_{l+j}}^{A_{\pi(l), s_{l+j}} \oplus C_{s_{l+j}}}\left\lceil u_{l+n+1}^{1}\right.\right. \\
& =\Theta_{\pi(l), 1, s_{l+n+1}}^{A_{\pi(l), s_{l+n+1}} \oplus C_{s_{l+n+1}}}\left\lceil u_{l+n+1}^{1}=E_{\pi(l), s_{l+\pi+1}}^{1}\left\lceil u_{l+n+1}^{1} .\right.\right.
\end{aligned}
$$

For (d), we have first by (a) for $j=(n-2)$,

$$
E_{\pi(l), s_{l+n-2}}^{3}\left\lceil u_{l+n-2}^{3} \neq E_{\pi(l), s_{l+n-1}}^{3}\left\lceil u_{l+n-2}^{3},\right.\right.
$$

so by $(1.2)(\mathrm{b})$, for some $1 \leq h \leq 3$,

$$
F_{\pi(l), s_{l+n-2}}^{h}\left\lceil w_{l+n-2}^{3} \subset F_{\pi(l), s_{l+n-1}}^{h} \uparrow w_{l+n-2}^{3} .\right.
$$

On the other hand, by (5)(b),

$$
F_{\pi(l), s_{i+n-2}}^{h}\left\lceil w_{l+n-2}^{3}=F_{\pi(l), s_{i+n+1}}^{h}\left\lceil w_{l+n-2}^{3},\right.\right.
$$

whence as before

$$
E_{\pi(l), s_{l+n-1}}^{2}\left\lceil u_{l+n-1}^{2} \neq E_{\pi(l), s_{l+n+1}}^{2}\left\lceil u_{l+n-1}^{2} .\right.\right.
$$

Thus by (c), $t=s_{l+n+1}$ satisfies the condition in square brackets and $t_{l}$ exists.

Towards a contradiction, suppose that $t_{l} \leq s_{l+n}$. If

$$
E_{\pi(l), t_{l}}^{1}\left\lceil u_{l+n-1}^{1} \neq E_{\pi(l), s_{l+n-1}}^{1}\left\lceil u_{l+n-1}^{1},\right.\right.
$$

then also $E_{\pi(l), s_{l+n}}^{1}\left\lceil u_{l+n-1}^{1} \neq E_{\pi(l), s_{l+n-1}}^{1}\left\lceil u_{l+n-1}^{1}\right.\right.$, contrary to (b). Hence the $E_{\pi(l)}^{1}$ parts agree and by (1.2)(a),

$$
F_{\pi(l), s_{l+n-1}}^{2}\left\lceilw _ { l + n - 1 } ^ { 2 } \subset F _ { \pi ( l ) , t _ { l } } ^ { 2 } \left\lceil w_{l+n-1}^{2} .\right.\right.
$$

But by the third condition of the definition of $t_{l}$,

$$
E_{\pi(l), t_{l}}^{1}\left\lceil u_{l+n-1}^{1}=E_{\pi(l), s_{l+n}}^{1}\left\lceil u_{l+n-1}^{1},\right.\right.
$$

so also $F_{\pi(l), s_{l+n-1}}^{2}\left\lceil w_{l+n-1}^{2} \subset F_{\pi(l), s_{l+n}}^{2}\left\lceil w_{l+n-1}^{2}\right.\right.$, contrary to (b).

Now (1.3) is proved exactly as for Theorem 1.2 and it remains to show (1.4) Only finitely many $s_{l}$ exist; in other words, $\lim \inf _{s} \rho(s)<\infty$. 
Proof. Suppose to the contrary that all $s_{l}$ exist. Then clearly the function $l \mapsto s_{l}$ is recursive in $C$, so by $6(\mathrm{c})$ we have $B \oplus E_{0}^{1} \oplus \cdots \oplus E_{n+1}^{1} \leq_{T} C$. By (4),

$$
s_{l+n}=\text { least } s<t_{l}\left[\phi\left(s, x_{l+n}\right) \wedge C_{s} \uparrow v\left(s, x_{l+n}\right)=C_{t_{l}}\left\lceil v\left(s, x_{l+n}\right)\right]\right.
$$

so $s_{l+n}$ can be calculated from $t_{l}$ and hence by $6(\mathrm{~d})$ from $s_{l+n-1}$ using $E_{\pi(l)}^{1}$ as an oracle. It follows that the function $l \mapsto s_{l}$ and hence $C$ is recursive in $B \oplus E_{0}^{1} \oplus \cdots \oplus$ $E_{n+1}^{1}$. Thus $C \equiv_{T} B \oplus E_{0}^{1} \oplus \cdots \oplus E_{n+1}^{1}$ so is of r.e. degree, contrary to hypothesis.

In combining the strategies for all the requirements (1), there are several new problems, and we must modify the basic module. We use a priority tree $T=\omega^{<\omega}$ and assign to each $\alpha \in T$ of length $e$ a strategy $\sigma_{\alpha}$ for satisfying the $e$-th requirement $(1)_{e}$ in some fixed listing. The nodes are ordered in the usual way:

$$
\alpha \leq \beta \quad \alpha \subseteq \beta \vee \exists e[\alpha \uparrow e=\beta \uparrow e \wedge \alpha(e)<\beta(e)]
$$

For each $\alpha$ which is active (to be defined in the construction) at stage $s$ we will define numbers $\rho_{\alpha}(s), x_{\alpha \frown\langle j\rangle}(s)$ for $j \leq \rho_{\alpha}(s)$, and $u_{\alpha \frown\langle j\rangle}^{i}(s), v_{\alpha \frown\langle j\rangle}(s)$, and $w_{\alpha \frown\langle j\rangle}^{i}(s)$ for $j<\rho_{\alpha}(s)$ and $1 \leq i \leq n$, which will play the same role in the action of $\sigma_{\alpha}$ as their counterparts do above. Strategy $\sigma_{\alpha}$ acts under the assumption that for all $e<|\alpha|$, $\sigma_{\alpha \mid e}$ has outcome $\alpha(e)$ - that is, $\liminf _{s} \rho_{\alpha \mid e}(s)=\alpha(e)$ - and only at those stages, called $\alpha$-stages, when this assumption is predicted by the evidence gathered to that point in the construction. At each stage $s$ we compute a sequence $\tau(s) \in T$ with $|\tau(s)|=s$ which represents our current prediction of the eventual outcome of the first $s$-many requirements. At stage $s+1$, if $\alpha=\tau(s+1) \uparrow e$, then $\tau(s+1)(e)$ will be the value of $\rho_{\alpha}(s+1)$ computed essentially as above: either $\rho_{\alpha}(s+1)=h<\rho_{\alpha}(s)$ because $c_{s+1}<v_{\alpha \sim\langle h\rangle}(s)$ for some (least) $h<\rho_{\alpha}(s)$, or $\rho_{\alpha}(s+1)=\rho_{\alpha}(s)+1$ because a new agreement is verified at $s$, or neither of these holds and $\rho_{\alpha}(s+1)=\rho_{\alpha}(s)$. A stage $s$ is an $\alpha$-stage iff $\alpha \subseteq \tau(s)$. Then we shall show that these values determine a true path $f$ defined by

$$
f(e)=\liminf _{s}\{\tau(s)(e): \tau(s)\lceil e=f\lceil e\}
$$

and that $\sigma_{f \mid e}$ satisfies the $e$-th requirement. More precisely, we will prove the following two assertions.

(1.5) If $C$ is not of r.e. degree, then there exists a path $f$ through $T$ such that for all $e$, there exists $\bar{s}$ such that if $\alpha$ denotes $f\lceil e$, then for all $s \geq \bar{s}$,

(a) $\bar{s}$ is an $\alpha$-stage;

(b) $\alpha \leq \tau(s)$ and $\alpha$ is active at $s$;

(c) if $s$ is an $\alpha$-stage, then for all $\beta \leq \alpha, x_{\beta}(s)$ is defined iff $x_{\beta}(\bar{s})$ is defined, in which case $x_{\beta}(s)=x_{\beta}(\bar{s})$ (which we denote by $x_{\beta}$ ), and for $k<(n+2)$ and all $t$, $A_{k, s}\left(x_{\beta}(t)\right)=A_{k, \bar{s}}\left(x_{\beta}(t)\right)$;

(d) if $s$ is $C$-true, then $s$ is an $\alpha$-stage.

Note that (b) and (d) together strengthen (*).

(1.6) For all $e$, if $(1)_{e}$ fails, then $\liminf _{s} \rho_{f \mid e}(s)=\infty$.

As is usual in tree arguments, we need to take care that any actions taken at nodes $\beta<\tau(s+1)$ are preserved at stage $s+1$. Threats to such actions are of two sorts: (i) new enumerations of elements into some $A_{k}$ below the use $v_{\beta}(s)$ of the agreement established at some earlier stage at $\beta$, and (ii) changes in $C$ which cause elements to 
be removed from some $A_{k}$. Problem (i) will be handled simply by choosing witnesses large enough to ensure that

$$
x_{\alpha}(s) \geq \max \left\{v_{\beta}(s): \beta<_{L} \alpha \wedge v_{\beta}(s) \text { is defined }\right\} .
$$

We note that $v_{\beta}(s+1)$ is undefined for $\beta \subseteq \tau(s+1)$.

Problem (ii) will be handled by ensuring that the uses of elements enumerated into $A_{k}$ at node $\alpha$ and stage $s+1$, which we shall denote by $p_{\alpha}(s+1)$, are chosen (possibly larger than $v_{\alpha}(s)$ ) in such a way that if $c_{s+1}<p_{\alpha}(s)$, then $\tau(s+1) \leq \alpha$. These two facts are stated formally as (2)(b) and (c) below.

The full construction now goes as follows. At stage 0 only $\emptyset$ is active, $\rho_{\emptyset}(0)=0$, and $x_{\langle 0\rangle}(0)=0$. At stage $s+1$ we proceed by induction on $e \leq s$. Any parameter not mentioned is assigned the same value at $s+1$ as at $s$. Let $\alpha=\tau(s+1) \uparrow e$; there are four cases. We write $\phi_{e}$ for the version of $\phi$ corresponding to $(1)_{e}$, but to prevent further degradation of readability, we will not attach this subscript to any of the other parameters of $(1)_{e}$.

Case 1 . If $\alpha$ was not active at stage $s$, then set $\rho_{\alpha}(s+1):=0$ and choose $x_{\alpha-\langle 0\rangle}(s+1)$ greater than any number used so far;

Case 2. If $\rho_{\alpha}(s)>0$ and for some (least) $j<\rho_{\alpha}(s), c_{s+1}<p_{\alpha}-\langle j\rangle(s)$, then $\rho_{\alpha}(s+1):=$ $j ; p_{\beta}(s+1), u_{\beta}^{i}(s+1), v_{\beta}(s+1)$ and $w_{\beta}^{i}(s+1)(1 \leq i \leq n)$ are undefined for $\beta=\alpha \frown\langle j\rangle$ and all $\beta \geq \alpha \frown\langle j+1\rangle ; x_{\beta}(s+1)$ is undefined for all $\beta \geq \alpha \frown\langle j+1\rangle$;

Case 3. otherwise, if $\rho_{\alpha}(s)=l$, and $\phi_{e}\left(s, x_{\alpha \frown l l\rangle}(s)\right)$ holds with associated uses $u^{i}\left(s, x_{\alpha-\langle l\rangle}(s)\right), w^{i}\left(s, x_{\alpha-\langle l\rangle}(s)\right)$ and $v\left(s, x_{\alpha}-\langle l\rangle(s)\right) \leq c_{s+1}$, then

(a) $\rho_{\alpha}(s+1):=l+1, u_{\alpha \frown\langle l\rangle}^{i}(s+1)=u^{i}\left(s, x_{\alpha-\langle l\rangle}(s)\right), v_{\alpha-\langle l\rangle}(s+1)=v\left(s, x_{\alpha-\langle l\rangle}(s)\right)$,

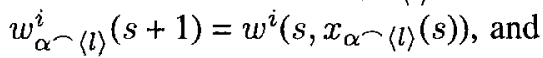

$$
p_{\alpha \frown\langle l\rangle}(s+1):=\max \left\{v_{\alpha \frown\langle l\rangle}(s+1)\right\} \cup\left\{p_{\beta}(s): \alpha^{\frown}\langle l\rangle \subseteq \beta \text { and } p_{\beta}(s) \text { is defined }\right\}
$$

(b) for $j<\min \{l, n-1\}, x_{\alpha-\langle l-j\rangle}(s)$ is enumerated into $A_{\pi(l-j), j, s+1}$ with use $p_{\alpha}-\langle l\rangle(s+1)$;

(c) if $l \geq n$, then $x_{a-\langle l-n\rangle}(s+1)$ is enumerated into $A_{\pi(l-n), n-1, s+1}$ with use $p_{\alpha} \frown\langle l\rangle(s+1)$;

(d) $x_{\alpha-\langle l+1\rangle}$ is chosen greater than any number used so far;

Case 4. otherwise $\rho_{\alpha}(s+1)=\rho_{\alpha}(s)$.

Set $\tau(s+1)(e)=\rho_{\alpha}(s+1)$. A node $\alpha$ is active at stage $s+1$ iff either $\alpha \subseteq \tau(s+1)$ or $\alpha<_{L} \tau(s+1)$ and $\alpha$ was active at stage $s$. In particular, if $\tau(s+1)<_{L} \alpha$, then $\alpha$ is inactive at stage $s+1$. If $\beta=\gamma \frown\langle j\rangle$, we write $\pi(\beta)$ for $\pi(j)$ and $\beta^{+}$for $\gamma\langle\langle j+1\rangle$. We have first:

(2) All parameters corresponding to inactive nodes are undefined. For all $s$, all $\alpha$ active at $s$, and all $\beta, 1 \leq i \leq n$, and $j$,

(a) $x_{\alpha \frown\langle j\rangle}(s)$ is defined for (exactly) $j \leq \rho_{\alpha}(s) ; \quad p_{\alpha \frown\langle j\rangle}(s), u_{\alpha \frown\langle j\rangle}^{i}(s), v_{\alpha \frown\langle j\rangle}(s)$, and $w_{\alpha \frown\langle j\rangle}^{i}(s)$ are defined for (exactly) all $j \leq \rho_{\alpha}(s)-1$;

(b) $\beta<_{L} \alpha \Longrightarrow p_{\beta}(s) \leq x_{\alpha}(s)$ (when both are defined);

(c) $\beta<_{L} \tau(s+1) \Longrightarrow p_{\beta}(s) \leq c_{s+1}$;

(d) for $j<(n-1)$ and $h<\rho_{\alpha}(s)-j, \quad x_{\alpha-\langle h\rangle}(s) \in A_{\pi(h), j, s}$ with use $\leq c_{s}$, but for $\rho_{\alpha}(s)-j \leq h \leq \rho_{\alpha}(s), \quad x_{\alpha} \sim\langle h\rangle \notin A_{\pi(h), \dot{j}, s}$; 
(e) for $h<\rho_{\alpha}(s)-n, \quad x_{\alpha-\langle h\rangle}(s) \in A_{\pi(h), n-1, s}$ with use $\leq c_{s}$, but for $\rho_{\alpha}(s)-n \leq$ $h \leq \rho_{\alpha}(s), \quad x_{\alpha-\langle h)}(s) \notin A_{\pi(h), n-1, s} ;$

(f) for all $t<s$, if $x_{\beta}(t)$ is defined but $x_{\beta}(s)$ is either undefined or $\neq x_{\beta}(t)$, then for all $s^{\prime} \geq s$ and $j<n, x_{\beta}(t) \notin A_{\pi(\beta), j, s^{\prime}}$. In particular, this holds when $x_{\beta}(t)$ is defined and $\tau(s)<_{L} \beta$.

Proof. For (a), by induction, note that for $\alpha<_{L} \tau(s+1)$, no changes are made in any of the $\alpha$-parameters at $s+1$, while for $\alpha \subseteq \tau(s+1)$, the construction ensures exactly these definitions. For (b), if $\beta<_{L} \alpha$, then $p_{\beta}(s)=p_{\beta}(t)$ for some $\beta$-stage $t<s$ for which $x_{\alpha}(t)$ is undefined. Hence when $x_{\alpha}(s)$ is defined, it is chosen larger than $p_{\beta}(s)$.

Next note that when both are defined we have

$$
\alpha \subseteq \beta \quad \Longrightarrow \quad p_{\beta}(s) \leq p_{\alpha}(s) .
$$

This inequality is true at the stage $s$ at which $p_{\alpha}(s)$ is defined by case 2 . Any change in the relevant $p_{\beta}$ occurs only at an $\alpha$-stage $t$ for which $p_{\alpha}(t)$ is undefined, so the change will be incorporated into the next definition of $p_{\alpha}$, if any. Now, for (c), suppose that $\beta<_{L} \tau(s+1)$, so for some $\gamma$ and $i \leq k, \gamma \frown\langle i\rangle \subseteq \beta$ and $\gamma-\langle k+1\rangle \subseteq \tau(s+1)$. Then by the construction and $(*)$,

$$
p_{\beta}(s) \leq p_{\gamma \frown\langle i\rangle}(s) \leq p_{\gamma} \frown\langle k\rangle(s) \leq c_{s+1} .
$$

Parts (d) and (e) hold as in the single requirement case using (c). For (f), suppose that $x_{\beta}(t)$ is defined and is enumerated into to $A_{\pi(\beta), j, t}$ with use $p_{\beta}(t)$ (or $p_{\beta^{+}}(t)$ ) but becomes undefined at some $t^{\prime}+1$ with $t \leq t^{\prime}<s$. Then by the construction, there exists some $\gamma$ and $h^{\prime}<h$ such that $\gamma \frown\left\langle h^{\prime}\right\rangle \subseteq \tau\left(t^{\prime}\right), \gamma-\langle h\rangle \subseteq \beta$ and

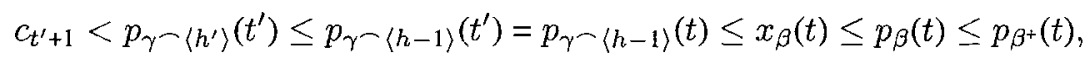

so $x_{\beta}(t)$ is removed from $A_{\pi(\beta), j}$ at stage $t^{\prime}+1$, unless this already happened at an earlier stage.

We begin now the proof of (1.5). Suppose that $\alpha=f\lceil e$ has been defined to satisfy (1.5); we aim to calculate $f(e)$ so that $f \uparrow(e+1)$ also satisfies (1.5). By (1.5)(b), for all $s \geq \bar{s}, \rho_{\alpha}(s)$ is defined. For each $l \geq-1$ for which it exists, let

$$
s_{\alpha-\langle l\rangle}=\text { least } s \geq \bar{s}(\forall t>s)\left[\rho_{\alpha}(t) \geq l+1\right] .
$$

Clearly when it exists, $s_{\alpha}-\langle l\rangle$ is an $\alpha$-stage. The proof follows closely the pattern of the proof of (1.4) above and we use the same numbering for the lemmas.

(3) For each $l \geq 0$, if $s_{\alpha-\langle l\rangle}$ exists, then,

(a) $\rho_{\alpha}\left(s_{\alpha-\langle l\rangle}\right)=l, \quad \phi_{e}\left(s_{\alpha \sim\langle l\rangle}, x_{\alpha-\langle l\rangle}\right), \quad$ and $\rho_{\alpha}\left(s_{\alpha-\langle l\rangle}+1\right)=l+1$;

(b) for all $s>s_{\alpha-\langle l\rangle}, \quad \rho_{\alpha}(s) \geq l+1, \alpha^{\frown}\langle l+1\rangle \leq \tau(s)$ and $\alpha \frown\langle l+1\rangle$ is active at stage $s$;

(c) for all $j \leq l, u_{\alpha \frown\langle j\rangle}^{i}(t), v_{\alpha \frown\langle j\rangle}(t), w_{\alpha \frown\langle j\rangle}^{i}(t), p_{\alpha \frown\langle j\rangle}(t)$, and $x_{\alpha-\langle j+1\rangle}(t)$ have the same values for all $t>s_{\alpha \frown\langle k\rangle}$ (which we denote by $u_{\alpha \frown\langle j\rangle}^{i}, v_{\alpha \frown\langle j\rangle}, w_{\alpha \frown\langle j\rangle}^{i}$, $p_{\alpha-\langle j\rangle}$ and $\left.x_{\alpha-\langle j+1\rangle}\right)$;

(d) $C_{s_{\alpha-\langle l\rangle}}\left|p_{\alpha-\langle l\rangle}=C\right| p_{\alpha-\langle l\rangle}$;

(e) for all $s \geq s_{\alpha}-\langle l\rangle$,

(i) for all $j<n-1$ and all $h \leq(l-j-1), \quad x_{\alpha-\langle h\rangle} \in A_{\pi(h), j, s}$;

(ii) for all $h \leq(l-n-1), \quad x_{\alpha \sim\langle h\rangle} \in A_{\pi(h), n-1, s}$; 
(f) for all $s>s_{\alpha}-\langle l\rangle$, $A_{\pi(l-j), j, s}$

(i) for all $j<(n-1), \quad x_{\alpha \sim\langle l-j\rangle} \notin A_{\pi(l-j), j, s_{\alpha} \sim\langle l\rangle}, \quad$ but $\quad x_{\alpha-\langle l-j\rangle} \in$

(ii) $x_{\alpha-\langle l-n\rangle} \notin A_{\pi(l-n), n-1, s_{\alpha-\langle l\rangle}}$, but $x_{\alpha \frown\langle l-n\rangle} \in A_{\pi(l-n), n-1, s}$;

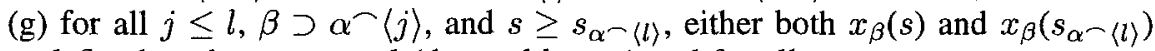
are undefined or they are equal (denoted by $x_{\beta}$ ) and for all $g<n$,

$$
A_{\pi(\beta), g, s}\left(x_{\beta}\right)=A_{\pi(\beta), g, s_{\alpha} \frown(l)}\left(x_{\beta}\right) .
$$

Proof. Part (a) follows as before and (b) follows from (1.5)(b) and the definition of $s_{\alpha \sim\langle l\rangle}$. Using (2)(c) we can repeat the earlier argument for (c) and (d), since by (b), $\alpha \smile\langle l\rangle<_{L} \tau(t)$. Parts (e) and (f) are proved as before. For (g), fix $j \leq l$ and $\beta \supset \alpha \frown\langle j\rangle$. Then for all $s>s_{\alpha-\langle l\rangle}, \beta<_{L} \tau(s)$, so $s$ is not a $\beta$-stage, and $x_{\beta}$ is neither defined nor undefined at stage $s$ so maintains its status and value. Likewise, $x_{\beta}:=x_{\beta}(s)$ will not be enumerated into any $A_{\pi(\beta), g}$ at stage $s$. Suppose that for some $g<n, x_{\beta} \in A_{\pi(\beta), g, s_{\alpha-\langle l)}}$ with use $p_{\beta}\left(s_{\alpha-\langle l\rangle}\right)$ (or $\left.p_{\beta^{+}}\left(s_{\alpha-\langle l\rangle}\right)\right)$. Then we can prove by induction on $s \geq s_{\alpha} \sim\langle l\rangle$ that also $p_{\beta}(s)=p_{\beta}\left(s_{\alpha}-\langle l\rangle\right)$ and $x_{\beta} \in A_{\pi(\beta), g, s}$ - the induction step uses (2)(c) (and the fact that also $\beta^{+}<_{L} \tau(s)$ ).

We have also the converse to (3)(d)

(4) For any $l$ and $s>\vec{s}$, if $\rho_{\alpha}(s) \geq l+1$ and $C_{s} \uparrow p_{\alpha \frown\langle l\rangle}(s)=C \uparrow p_{\alpha \frown\langle l\rangle}(s)$, then $s_{\alpha} \frown\langle l\rangle$ exists and $s>s_{\alpha}-\langle l\rangle$.

Proof. As for a single requirement.

(5) For each $l \geq 0$, if $s_{\alpha-\langle l+n+1\rangle}$ exists, then

(a) for all $j<(n-2), \quad A_{\pi(l), s_{\alpha \frown\langle l+j)}} \mid v_{\alpha \frown\langle l+j\rangle}=A_{\pi(l), s_{x} \frown\langle l+j+2)}\left\lceil v_{\alpha \frown\langle l+j\rangle}\right.$;

(b) $A_{\pi(l), s_{\alpha \frown\langle l+n-2\rangle}}\left\lceil v_{\alpha \frown\langle l+n-2\rangle}=A_{\pi(l), s_{\alpha \frown\langle l+n+1)}}\left\lceil v_{\alpha \frown\langle l+n-2\rangle}\right.\right.$;

(c) $A_{\pi(l), s_{\alpha} \backslash\langle l+n-1)} \mid v_{\alpha \smile\langle l+n-1\rangle}=A_{\pi(l), s_{\alpha} \sim(l+n)} \uparrow v_{\alpha \sim\langle l+n-1\rangle}$.

Proof. Consider first (a) and fix $j<(n-2)$. It suffices to verify that for all $\beta$ and all $t \leq s_{\alpha \frown\langle l+j+2\rangle}$ such that $x_{\beta}(t)<p_{\alpha \smile\langle l+j\rangle}$,

$$
A_{\pi\left\{(l), s_{\alpha}-\{l+j\}\right.}\left(x_{\beta}(t)\right)=A_{\pi(b), s_{\alpha}-\langle l+j+2\}}\left(x_{\beta}(t)\right) .
$$

For $\beta$ such that $\alpha \smile\langle l+j+2\rangle<_{L} \beta$ this follows from (2)(f): for all $g<n$ and $s=$ $s_{\alpha \frown\langle l+j\rangle}$ or $s=s_{\alpha \smile\langle l+j+2\rangle}, x_{\beta}(t) \notin A_{\pi(l), g, s}$. If $\alpha \frown\langle l+j+1\rangle \subseteq \beta$ or $\alpha \frown\langle l+j+2\rangle \subseteq \beta$, the same argument applies for $t \leq s_{\alpha} \frown\langle l+j\rangle$, while if $s_{\alpha \frown\langle l+j\rangle}<t \leq s_{\alpha}-\langle l+j+2\rangle$, we have $p_{\alpha \frown\langle l+j\rangle} \leq x_{\beta}(t)$ by (2)(b). If $\alpha \frown\langle h\rangle \subset \beta$ for some $h \leq l+j$, we have by (2)(f) (for $\left.t \leq s_{\alpha-\langle l+j\rangle}\right)$ and (3)(g) that for all $g<n$ and $s \geq s_{\alpha-\langle l+j\rangle}$,

$$
A_{\pi(l), g, s}\left(x_{\beta}(t)\right)=A_{\pi(l), g, s_{\alpha} \frown\langle l+j\rangle}\left(x_{\beta}(t)\right) .
$$

If $\beta \leq \alpha$, the result follows from (1.5)(c) of the induction hypothesis. If $\beta=\alpha \frown\langle h\rangle$ for some $h \leq l+j$ and $x_{\beta}(t) \neq x_{\beta}\left(s_{\alpha}-\langle l\rangle\right)$, then we may again apply (2)(f). We are left with the numbers $x_{\alpha} \sim\langle h\rangle$ for $h \leq l+j$ with $\pi(h)=\pi(l)$. By (3)(e) and (f), for such $h$,

$$
\begin{gathered}
h \leq l-(n+2) \quad \Longrightarrow \quad(\forall g<n)\left(\forall s \geq s_{\alpha \sim\langle l+j\rangle}\right)\left[x_{\alpha \sim\langle h\rangle} \in A_{\pi(l), g, s}\right] \\
x_{\alpha \sim\langle l\rangle} \in A_{\pi(l), g, s_{\alpha \sim\langle l+j\rangle}} \quad \text { for exactly } g=0,1, \ldots, j-1 \\
x_{\alpha \frown\langle l\rangle} \in A_{\pi(l), g, s_{\alpha \sim\langle l+j+2\rangle}} \quad \text { for exactly } g=0,1, \ldots, j+1
\end{gathered}
$$


Thus the result follows as above. We leave the similar proofs of (b) and (c) to the devoted reader.

(6) For each $l>0$,

(a) if $s_{\alpha-\langle l+n+1\rangle}$ exists, then for all $j \leq(n-2)$,

$$
E_{\pi(l), s_{\alpha} \frown\langle l+j\rangle}^{n-j+1}\left\lceil u_{\alpha \smile\langle l+j\rangle}^{n-j+1} \neq E_{\pi(l), s_{\alpha}-\langle l+j+1\rangle}^{n-j+1} \uparrow u_{\alpha-\langle l+j\rangle}^{n-j+1}\right.
$$

(b) if $s_{\alpha-\langle l+n\rangle}$ exists, then $E_{\pi(l), s_{\alpha} \backslash\langle l+n-1\rangle}^{2}\left|u_{\alpha-\langle l+n-1\rangle}^{2}=E_{\pi(l), s_{\alpha \sim\langle l+n\rangle}}^{2}\right|$ $u_{\alpha-\langle l+n-1\rangle}^{2}$;

(c) if $s_{\alpha \smile\langle l+j\rangle}$ exists for all $j$, then $E_{\pi(l), s_{\alpha}-\langle l+n+1\rangle}^{1}\left\lceil u_{\alpha^{-}\langle l+n+1\rangle}^{1}=E_{\pi(l)}^{1} \uparrow\right.$ $u_{\alpha \sim\langle l+n+1\rangle}^{1}$;

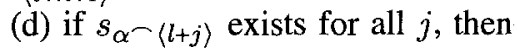

$$
\begin{aligned}
& \exists t>s_{\alpha \frown\langle l+n-1\rangle}\left[\phi\left(t, x_{\alpha \frown\langle l+n-1\rangle}\right) \wedge E_{\pi(l), t}^{2} \mid u_{\alpha-\langle l+n-1\rangle}^{2}\right. \\
& \quad \neq E_{\pi(l), s_{\alpha} \frown\langle l+n-1\rangle}^{2}\left\lceil u_{\alpha \frown\langle l+n-1\rangle}^{2}\right. \\
& \quad \wedge E_{\pi(l), t}^{1} \mid u^{1}\left(t, x_{\alpha-\langle l+n-1\rangle}\right)=E_{\pi(l)}^{1}\left\lceil u^{1}\left(t, x_{\alpha \frown\langle l+n-1\rangle}\right)\right],
\end{aligned}
$$

and if $t_{l}$ denotes the least such $t$, then $s_{\alpha \frown\langle l+n\rangle}<t_{l}$.

Proof. The computations here exactly mirror those of the corresponding part of the single-requirement case, with each subscript $l+\cdots$ replaced by $\alpha \frown\langle l+\cdots\rangle$ and using the $\alpha$-versions of (4) and (5).

(1.4) Only finitely many $s_{\alpha \sim\langle l\rangle}$ exist; in other words, $\lim \inf _{s} \rho_{\alpha}(s)<\infty$.

Proof. Exactly as before, under the assumption that for all $l, s_{\alpha} \frown\langle l\rangle$ exists, we may derive from (4) and (6) that $C$ is of r.e. degree, contrary to hypothesis.

Now we may verify (1.5) for $e+1$ as follows. Set $f(e)=l:=\liminf _{s} \rho_{\alpha}(s)$, $\alpha^{+}=f \mid(e+1):=\alpha^{-}\langle l\rangle$, and $s^{+}=s_{\alpha-\langle l-1\rangle}+1$. By (3)(a), $s^{+}$is an $\alpha^{+}$-stage. For $s \geq s^{+}$either $\alpha<_{L} \tau(s)$ or $\alpha \subseteq \tau(s)$, so $\alpha^{-}\langle l\rangle \leq \tau(s)$ by (3)(b). In either case, $\alpha^{+} \leq \tau(s)$ as required by (1.5)(b). For (c), let $s \geq s^{+}$be an $\alpha^{+}$-stage and $\beta \leq \alpha^{+}$. The new cases to verify are $\alpha-\langle j\rangle \subseteq \beta$ for $j<l$ and $\beta=\alpha^{+}$. The first clause (concerning $x_{\beta}(s)$ ) follows from (3)(g) when $\alpha^{-}\langle j\rangle \subset \beta$ and $j<l$ and from (3)(c) when $\alpha \frown\langle j\rangle=\beta$ and $j \leq l$. The second clause (concerning $A_{k, s}\left(x_{\beta}(t)\right.$ )) follows from (2)(f) for $t<s^{+}$and all $\beta$, from (3)(g) when $t>s^{+}, \alpha \frown\langle j\rangle \subset \beta$, and $j<l$, and from (2)(d)(e) when $t>s^{+}, \alpha^{-}\langle j\rangle=\beta$ and $j \leq l$. Finally, let $s \geq s^{+}$be a $C$-true stage. Then $\alpha \subseteq \tau(s)$, so for some $l^{\prime} \geq l$, $\alpha \subsetneq\left\langle l^{\prime}\right\rangle \subseteq \tau(s)$. Suppose that $l^{\prime}>l$ so $\alpha \neg\langle l\rangle<_{L} \tau(s)$. Then by (2)(c), $p_{\alpha-\langle l\rangle}(s-1) \leq c_{s}$, so since $s$ is $C$ true, $C_{s}\left\lceil p_{\alpha-\langle l\rangle}(s-1)=C\left\lceil p_{\alpha \sim\langle l\rangle}(s-1)\right.\right.$. But then by (4), $s_{\alpha-\langle l\rangle}$ exists and $\lim \inf _{s} \rho_{\alpha}(s) \geq l+1$, contrary to the choice of $l$. Hence $\alpha^{+} \subseteq \tau(s)$ and $s$ is an $\alpha^{+}$-stage.

Finally, (1.6) follows as in each of the other cases.

In conclusion, we want to explain the difficulty in extending this proof to cover cases of the Conjecture for $m \geq 2$. We return to the informal description preceding the proof and attempt to adapt it to the case $(2,1)$ - that is, given $C$ which is 3-REA but not of 2-REA degree, we want to find an r.e.[C] set $A$ such that $A \oplus C$ is not of 3REA degree. Since $n=1$, the construction will resemble closely that of Theorem 1.2 except that we construct three sets $A_{0}, A_{1}$, and $A_{2}$. Assuming that $C_{s_{l}} \uparrow v_{l}=C\left\lceil v_{l}\right.$, 
we enumerate $x_{0}$ into $A_{0, s_{1}+1}$ with use $v_{1}, x_{1}$ into $A_{1, s_{2}+1}$ with use $v_{2}, x_{2}$ into $A_{2, s_{3}+1}$ with use $v_{3}$, etc. Since

$$
A_{0, s_{0}}\left(x_{0}\right)=A_{0, s_{1}}\left(x_{0}\right) \neq A_{0, s_{2}}\left(x_{0}\right)
$$

we have

$$
E_{0, s_{0}}^{3}\left\lceil u_{0}^{3}=E_{0, s_{1}}^{3}\left\lceil u_{0}^{3} \neq E_{0, s_{2}}^{3}\left\lceil u_{0}^{3}\right.\right.\right.
$$

and we may define

$t_{0}=$ least $t>s_{0}\left[\phi\left(t, x_{0}\right) \wedge E_{0, t}^{3}\left\lceil u_{0}^{3} \neq E_{0, s_{0}}^{3}\left\lceil u_{0}^{3} \wedge E_{0, t}^{2}\left\lceil u^{2}\left(t, x_{0}\right)=E_{0}^{2}\left\lceil u^{2}\left(t, x_{0}\right)\right]\right.\right.\right.\right.$.

If necessarily $s_{1} \leq t_{0}$, then we could compute $s_{1}$ (and by extension the entire function $l \mapsto s_{l}$ ) recursively in $B \oplus E_{0}^{2} \oplus E_{1}^{2} \oplus E_{2}^{2}$ and conclude that $C$ is Turing equivalent to this set and thus of 2-REA degree, contrary to hypothesis. Unfortunately, there seems to be no reason to expect that $s_{1} \leq t_{0}$. In the earlier case we had

$$
E_{0, s_{0}}^{2}\left\lceil u_{0}^{2}=E_{0, s_{1}}^{2}\left\lceilu _ { 0 } ^ { 2 } \text { and } E _ { 0 , s _ { 0 } } ^ { 2 } \left\lceilu _ { 0 } ^ { 2 } \subset E _ { 0 , t _ { 0 } } ^ { 2 } \left\lceil u_{0}^{2}\right.\right.\right.\right.
$$

Elements of $E_{0, t_{0}}^{2} \uparrow u_{0}^{2}$ are witnessed by $E_{0}^{1}$-correct axioms which never change, since $E_{0}^{1}$ is an r.e. set, and thus if $t_{0}<s_{1}$, the new elements could not be removed by stage $s_{1}$. Here, however, $E_{0, t_{0}}^{3}\left\lceil u_{0}^{3}\right.$ may differ from $E_{0, s_{0}}^{3}\left\lceil u_{0}^{3}\right.$ by both gaining and losing elements. If $t_{0}<s_{1}$, lost elements could be restored by stage $s_{1}$ and new elements could be removed; even though their axioms are eventually $E_{0}^{2}$-correct, since $E_{0}^{2}$ is only a 2-REA set, they may be temporarily unsatisfied at stage $s_{1}$. We see no way around this problem and expect that if the full conjecture is to be proved a quite different method will be needed.

\section{References}

[ChHi] Cholak, P.A., Hinman, P.G.: Iterated Relative Recursive Enumerability (abstract). Abstr. Am. Math. Soc. 881, 347 (1993)

[JoSh1] Jockusch, C.G., Shore, R.A.: Pseudo-jump operators. I; the r.e. case. Trans Amer. Math. Soc. 275, 599-609 (1983)

[JoSh2] Jockusch, C.G., Shore, R.A.: Pseudo-jump operators. II: Transfinite iterations, hierarchies and minimal covers. Jour. Symb. Log. 49, 1205-1236 (1984)

[SoSt] Soare, R.I., Stob, M.: Relative Recursive Enumerability. In: Stern, J. (ed.) Proceedings of the Herbrand Symposium: Logic Colloqium '81, pp. 299-324. Amsterdam: North-Holland 1982

[So] Soare, R.I.: Recursively enumerable sets and degrees, xviii+437 pp. Berlin Heidelberg: Springer 1987 